\title{
Distribution and conservation of three important bird groups of the Atlantic Forest in north-east Brazil
}

\author{
G. A. Pereira ${ }^{*}$, H. F. P. Araújo ${ }^{b}$ and S. M. Azevedo-Júnior ${ }^{a}$ \\ aPrograma de Pós-graduação em Etnobiologia e Conservação da Natureza, Laboratório de Ornitologia, \\ Departamento de Biologia, Universidade Federal Rural de Pernambuco - UFRPE, \\ Rua Dom Manuel de Medeiros, s/n, CEP 52171-900, Recife, PE, Brazil \\ ${ }^{\text {b}}$ Departamento de Ciências Biológicas, Centro de Ciências Biológicas, Universidade Federal da Paraíba - UFPB, \\ Campus Universitário, Rua Santa Rita, 130, CEP 58397-000, Areia, PB, Brazil \\ *e-mail: glaucoapereira@yahoo.com.br
}

Received: April 22, 2015 - Accepted: October 1, 2015 - Distributed: November 30, 2016

(With 4 figures)

\begin{abstract}
The Pernambuco Endemism Center in north-east Brazil has the most fragmented forest cover and the largest number of threatened birds of the whole Atlantic Forest. We analyzed the distribution of three groups of bird species: forest-dependent, endemic and/or threatened using the interpolation method of Inverse Distance Weighting. We also checked the concentration of these birds in protected and unprotected areas, suggesting new sites that need to be protected. The richness concentration of forest-dependent, endemic and/or threatened birds in 123 sites were analysed. There was a greater concentration of the three groups in north Alagoas, south and north Pernambuco, and north and west Paraíba. The distribution of the three groups was almost regular in different vegetation types, although a lower concentration was found in the pioneer formation. There was a greater concentration of birds from all three groups between Pernambuco and Alagoas, and this must be due to the presence of more forest fragments with better structure and vegetation heterogeneity. The protected and unprotected areas hosted important records of endemic and/or threatened birds. We suggested some important places for implementation of new protected areas due to the larger concentrations of the target birds and because they are located within the boundaries of the Important Bird Areas.
\end{abstract}

Keywords: neotropical birds, threatened birds, endemism, Atlantic Forest, conservation.

\section{Distribuição e conservação de três importantes grupos de aves na Floresta Atlântica do Nordeste do Brazil}

\begin{abstract}
Resumo
O Centro de Endemismo Pernambuco, situado no Nordeste do Brasil, é o setor mais fragmentado e com o maior número de aves ameaçadas de extinção de toda a Mata Atlântica. Foram analisadas as distribuições das aves florestais, endêmicas e/ou ameaçadas de extinção através do método da interpolação 'Inverse Distance Weighting'. Também foi verificada a concentração dessas aves em áreas protegidas e não protegidas, sugerindo novas áreas que necessitam ser protegidas. Foi verificada a concentração da riqueza das espécies de aves florestais, endêmicas e ameaçadas em 123 locais. Houve uma maior concentração dos três grupos no norte de Alagoas, sul e norte de Pernambuco, e norte e oeste da Paraíba. A distribuição dos três grupos foi regular nos distintos tipos vegetacionais, apesar de uma menor concentração nas áreas de formação pioneira. Houve maior concentração das aves dos três grupos entre Pernambuco e Alagoas, devido, provavelmente a presença de uma maior quantidade de fragmentos florestais com melhor estrutura e heterogeneidade vegetal. Tanto as áreas protegidas como as não protegidas possuem importantes registros de aves endêmicas e ameaçadas. Sugerimos alguns locais importantes para a implementação de novas áreas protegidas, tendo como base as áreas com maiores concentrações dos três grupos de aves e dentro dos limites das Important Bird Areas.
\end{abstract}

Palavras-chave: aves neotropicais, aves ameaçadas, endemismos, Mata Atlântica, conservação.

\section{Introduction}

The Brazilian Atlantic Forest in the states of Alagoas, Pernambuco, Paraíba and Rio Grande do Norte, known as Pernambuco Endemism Center (henceforth PEC)
(Silva et al., 2004) retains just only $11.45 \%$ of its original vegetation cover (SOSMA, 2015), and is currently one of the most degraded areas of the whole dominium, considered 
a hotspot within another hotspot (Tabarelli et al., 2006), the entire Atlantic Forest (Myers et al., 2000).

Despite the high level of fragmentation, 434 bird species persist in the region (Roda et al., 2011), which has the highest number of threatened birds in Brazil, according to the last updated list of threatened birds (Brasil, 2014; IUCN, 2015). Then, some research related to the conservation of threatened birds was carried out in the region, e.g. Pauxi mitu, Glaucidium mooreorum, Terenura sicki, Philydor novaesi, Tangara fastuosa, etc (Silveira et al., 2003b, 2008; Roda et al., 2011).

This current alarming situation of the PEC has occurred due to the removal of the native vegetation (Olmos, 2005; Silveira et al., 2003a). Currently there are only 157 protected areas in the region (Paula, 2012), and probably many of these are not functional for conservation of endemic and/or threatened birds, as noted by Giorgi et al. (2014) in southeastern Atlantic Forest. There are also the Important Bird Areas (IBA henceforth) which are places of great importance due to the significant number of endemic and/or threatened bird species, and they ensure the long-term conservation of these birds and areas (Bencke et al., 2006). Throughout the region there are 42 IBAs, many of which overlap protected areas.

The understanding of the biogeographic aspects of birds in fragmented areas is a challenge, given the adverse factors caused by fragmentation (Lees and Peres, 2008), such as the consequent loss of habitat that leads many species to local extinction, mainly those restricted to certain habitats (Moura et al., 2014). Thus to evaluate the application of biogeographic principles related to the dynamics of avian distribution is an extremely useful tool in conservation planning (Whittaker et al., 2005; Giorgi et al., 2014).

The distribution of a living being in a region can be checked through the methods of ecological modeling. The Inverse Distance Weight (IDW henceforth) interpolation modeling technique has been shown to be very effective due to its accuracy in providing a real picture of actual and potential distribution (see Roberts et al., 2004; Abdi and Nandipati, 2009). Besides, this method is very accessible, easy to use, and uses linear combinations of weights at known points to estimate unknown localities values (Roberts et al., 2004).

Therefore, the aims of this study were: 1) Analyze the distribution of forest-dependent, endemic and/or threatened birds along the entire length of the PEC using the IDW technique; 2) Verify how is the distribution of three groups of birds in the different vegetation types in the region; 3) Check the protected and unprotected areas that have higher concentrations of endemic and/or threatened birds; 4) suggest new areas within the limits of IBAs that could be turned into new protected areas according to the amount of endemic and/or threatened birds.

\section{Material and Methods}

\subsection{Study area}

The study was conducted in 123 sites of the Atlantic Forest of the Brazilian states of Alagoas, Pernambuco, Paraíba and Rio Grande do Norte, known as PEC. This sector is covered by five vegetation type: open ombrophilous forest, dense ombrophilous forest, ecological tension zone, seasonal semideciduous forest and pioneer formation (IBGE, 2004) (Figure 1). The altitude reaches more than $900 \mathrm{~m}$; the average temperature ranges from 24 to $26^{\circ} \mathrm{C}$, and the average annual rainfall is of $1,1180 \mathrm{~mm}$, with the rains being more consistent in the west and in the highlands (IBGE, 1985).

\subsection{Sampling}

The database used in this work was provenient from the literature, authors' fieldwork and records provided by other researchers. The target species are those dependent on forest environments (Roda, 2003, 2006), endemics of the PEC (Roda, 2003; Silveira et al., 2003a) and/or threatened birds mentioned in the Brazilian and global red list (Brasil, 2014; IUCN, 2015). The nomenclature of species follows the Brazilian Ornithological Records Committee (Piacentini et al., 2015).

\subsection{Data analysis}

We utilized the deterministic IDW interpolation modeling technique to determine areas with higher concentrations (densities) of the target species. This method determines cell values using a linear-weighted combination set of sample points. The weight assigned is a function of the distance of an input point from the output cell locations (Childs, 2004). Thereby, weights diminish as the distance increases from the point sampled (Childs, 2004; Roberts et al., 2004; Abdi and Nandipati, 2009).

We used Environmental System Research Institute's (ESRI) ArcGis 10.2.1 to create the density of the three bird groups' distribution maps through the data provided by IDW technique. An analysis of variance (ANOVA) and the Tukey post-hoc test were utilized to compare the species richness of forest-dependent, endemic and/or threatened birds with the different phytophysiogomies.

The occurrence of protected areas was consulted in the National Register of Protected Areas (http://www.mma.gov. $\mathrm{br} /$ areas-protegidas/cadastro-nacional-de-ucs), which is an updated database of the National System of Conservation Units (SNUC). The categories mentioned were: Biological Reserves (REBIO), State Parks (PE), Ecological Stations (ESEC), Municipal Parks (PM), Wildlife Refuges (RVS), Environmental Protection Area (APA), Private Reserves of Natural Heritage (RPPN) and Urban Forest Reserve (FURB) (SNUC, 2000). This last category was included in the system by law No. 14.324/2011 (Paula, 2012).

The IBAs of the northeastern Atlantic Forest cited in this work were those presented in Bencke et al. (2006).

\section{Results}

The species richness concentration of 171 forest-dependent, 26 endemic and 46 threatened birds were analysed (Table 1). The presence data of these bird groups were collected from 123 sites: 69 in Pernambuco, 40 in Alagoas, 11 in Paraíba and 03 in Rio Grande do Norte (see Figure 1). 


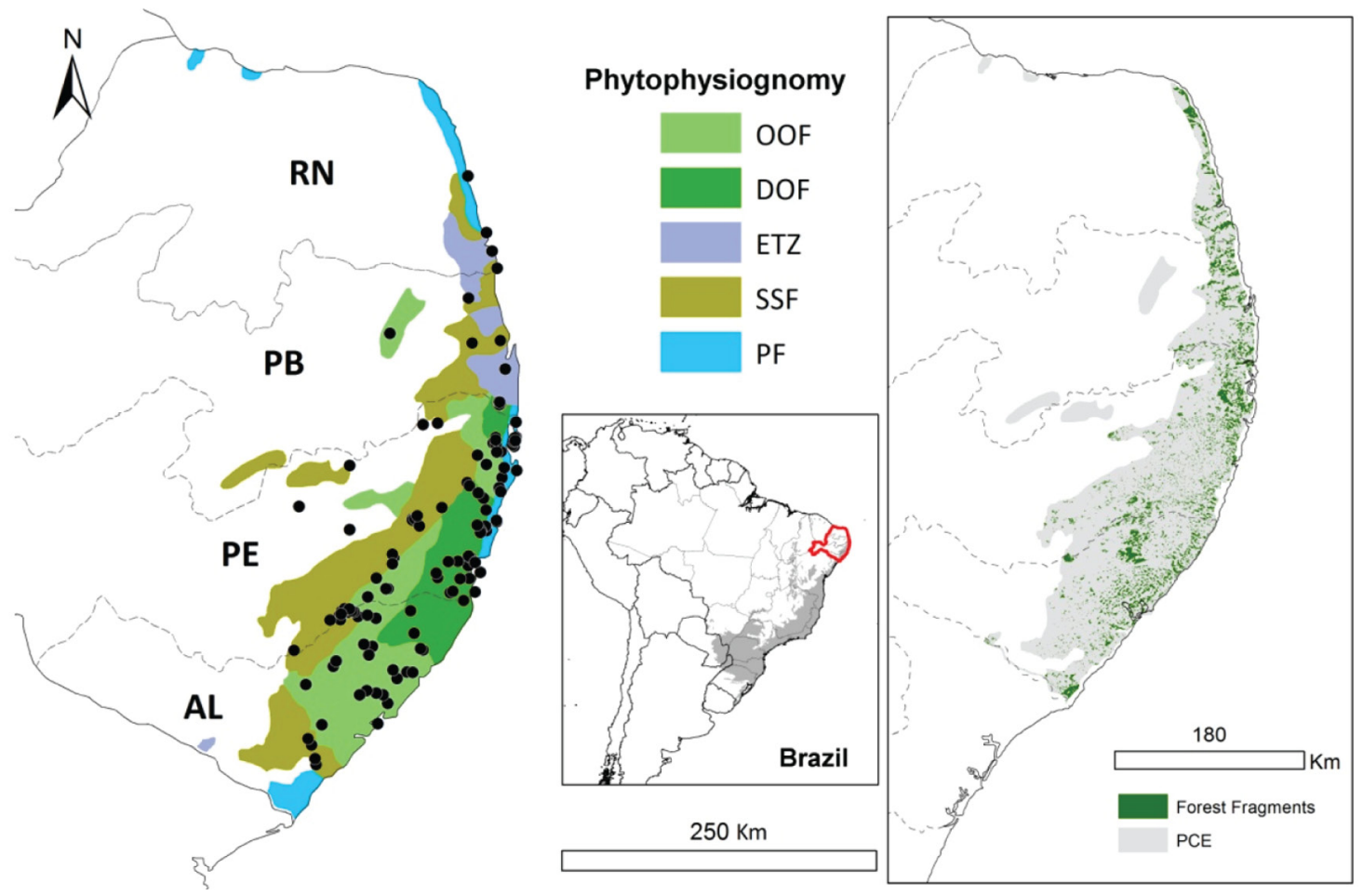

Figure 1. Localities (black dots) where bird surveys, vegetation types and forest fragments of the Pernambuco Endemism Center were conducted. Phytophysiognomies: OOF - Open Ombrophilous Forest; DOF - Dense Ombrophilous Forest; ETZ - Ecological Tension Zone; SSF - Stational Semideciduous Forest; and PF - Pioneer Formation.

The information of these sites, phytophysiognomies and richness of the three groups are listed in the Table 2.

The map created through the interpolation method showed greater concentration of forest-dependent birds between the states of Pernambuco and Alagoas, as well as some isolated spots in east and north Pernambuco and in north and west Paraíba (Figure 2a). The maps of the endemic and threatened birds showed similar patterns, but with less representation in the state of Paraíba. Moreover, all three groups of birds had very low densities in the state of Rio Grande do Norte (mainly in the far north) and in the south of the state of Alagoas (Figure 2b, c).

There was virtually no difference in the distribution of the species richness of the groups of birds between the phytophysiognomies, with the exception of the areas of pioneer formations (restingas), which presented lower species richness than all other formations (Figure 3; Table 3).

Only $32.5 \%$ of the forest fragments analysed was found into the boundaries of officially protected areas. The ESEC de Murici is the protected area with the highest number of species of the three bird groups, followed by RPPN Frei Caneca/RPPN Pedra D'Anta, REBIO de Saltinho and RPPN Eco Fazenda Morim (Figure 4a). The non-protected areas that had the largest concentrations of birds from all three groups were: Engenho Coimbra, Mata de Xanguá, Engenho Cachoeira Linda and Mata do Estado (Figure 4b). The protected and non-protected areas with the highest number of species of the three groups overlapped with the following IBAs: Murici, Serra do Urubu, Guadalupe, Engenho Coimbra, and Mata do Estado.

\section{Discussion}

The maps of the target birds made through the information generated by the IDW modeling technique were pretty accurate and showed higher densities in the areas between the states of Alagoas and Pernambuco. Roda et al. (2011) found a distribution more concentrated in that same area for seven endemic and threatened birds. This same pattern of distribution with highest densities of records between the states of Pernambuco and Alagoas was found for some species of forest raptors (Roda and Pereira, 2006).

As the richness concentration of the target species is distributed almost regularly in all vegetation types, then other variables must be acting on the distribution of these birds. It is known that the bird distribution occurs due to a set of several biotic and abiotic variables, and other factors such as movement and interaction (see Newton, 2003). Owing to the fact that the vegetation heterogeneity and complexity also contribute effectively to the species richness and distribution (Karr, 1990), then, this can be the answer to the great richness of bird groups in Pernambuco and Alagoas that have more quantities of forest fragments in later successional stages. Conversely, northern Paraíba and Rio Grande do Norte have low floristic diversity and are 
Table 1. List of the forest, endemic and/or threatened birds of the Pernambuco Endemism Center with their respective English names, vegetation types and threat categories. The names in bold represent the endemic birds of the Pernambuco Endemism Center.

\begin{tabular}{|c|c|c|c|c|}
\hline \multirow[t]{2}{*}{ Taxon } & \multirow[t]{2}{*}{ English Name } & \multirow[t]{2}{*}{ Vegetation type } & \multicolumn{2}{|c|}{$\begin{array}{l}\text { Category } \\
\text { of threat }\end{array}$} \\
\hline & & & МMA & IUCN \\
\hline Tinamus solitarius (Vieillot, 1819) & Solitary Tinamou & OOF & & \\
\hline Crypturellus soui (Hermann, 1783) & Little Tinamou & OOF,DOF,SSF,ETZ & & \\
\hline $\begin{array}{l}\text { Crypturellus strigulosus } \\
\text { (Temminck, 1815) }\end{array}$ & Brazilian Tinamou & OOF & & \\
\hline $\begin{array}{l}\text { Penelope superciliaris alagoensis } \\
\text { Nardelli, } 1993\end{array}$ & Rusty-margined Guan & OOF,DOF,SSF,ETZ,PF & CR & \\
\hline Ortalis araucuan (Spix, 1815) & East Brazilian Chachalaca & OOF,DOF,SSF,ETZ,PF & & \\
\hline $\begin{array}{l}\text { Odontophorus capueira plumbeicollis } \\
\text { Cory, } 1915\end{array}$ & Spot-winged Wood-quail & OOF,SSF,ETZ & CR & \\
\hline Leptodon forbesi (Swann, 1922) & White-collared Kite & OOF,DOF,SSF,ETZ & EN & $\mathrm{CR}$ \\
\hline $\begin{array}{l}\text { Chondrohierax uncinatus } \\
\text { (Temminck, 1822) }\end{array}$ & Hook-biled Kite & OOF,DOF,SSF,ETZ,PF & & \\
\hline Harpagus bidentatus (Latham, 1790) & Doubled-toothed Kite & OOF & & \\
\hline Harpagus diodon (Temminck, 1823) & Rufous-thighed Kite & DOF,ETZ & & \\
\hline Accipiter bicolor (Vieillot, 1817) & Bicolored Hawk & OOF,SSF ETZ & & \\
\hline Pseudastur polionotus (Kaup, 1847) & Mantled Hawk & OOF, DOF, SSF & & \\
\hline Buteo albonotatus Kaup, 1847 & Zone-tailed Hawk & OOF,DOF,SSF,ETZ,PF & & \\
\hline Spizaetus tyrannus (Wied, 1820) & Black Hawk-Eagle & OOF,SSF,ETZ & & \\
\hline Spizaetus melanoleucus (Vieillot, 1816) & $\begin{array}{c}\text { Black-and-White } \\
\text { Hawk-Eagle }\end{array}$ & SSF & & \\
\hline Patagioenas speciosa (Gmelin, 1789) & Scaled Pigeon & OOF,DOF,SSF,ETZ & & \\
\hline $\begin{array}{l}\text { Patagioenas cayennensis } \\
\text { (Bonnaterre, } 1792 \text { ) }\end{array}$ & Pale-vented Pigeon & OOF,DOF,SSF,ETZ,PF & & \\
\hline $\begin{array}{l}\text { Leptotila rufaxilla } \\
\text { (Richard \& Bernard, 1792) }\end{array}$ & Grey-fronted Dove & OOF,DOF,SSF,ETZ,PF & & \\
\hline Geotrygon violacea (Temminck, 1809) & Violaceous Quail-Dove & OOF & & \\
\hline Geotrygon montana (Linnaeus, 1758) & Ruddy Quail-Dove & OOF,DOF,SSF,ETZ & & \\
\hline $\begin{array}{l}\text { Megascops atricapilla } \\
\text { (Temminck, 1822) }\end{array}$ & Black-capped Screech-Owl & OOF & & \\
\hline Pulsatrix perspicillata (Latham, 1790) & Spectacled Owl & OOF,DOF,SSF,ETZ,PF & & \\
\hline Strix virgata (Cassin, 1849) & Mottled Owl & OOF,DOF,SSF,ETZ & & \\
\hline $\begin{array}{l}\text { Glaucidium mooreorum } \\
\text { (Silva, Coelho \& Gonzaga, 2002) }\end{array}$ & Pernambuco Pygmy-Owl & DOF & EX & $\mathrm{CR}$ \\
\hline Nyctiphrynus ocellatus (Tschudi, 1844) & Ocellated Poorwill & OOF,DOF,SSF,ETZ & & \\
\hline Lurocalis semitorquatus (Gmelin, 1789) & Short-tailed Nighthawk & OOF,DOF,SSF,ETZ & & \\
\hline Glaucis hisurtus (Gmelin, 1788) & Rufous-breasted Hermit & OOF,DOF,SSF,ETZ & & \\
\hline Phaethornis ruber (Linnaeus, 1758) & Reddish Hermit & OOF,DOF,SSF,ETZ,PF & & \\
\hline $\begin{array}{l}\text { Phaethornis margarettae camargoi } \\
\text { (Grantsau, 1988) }\end{array}$ & Margaretta’s Hermit & OOF,DOF,SSF & CR & \\
\hline Florisuga fusca (Vieillot, 1817) & Black Jacobin & OOF,DOF,SSF,ETZ,PF & & \\
\hline Lophornis magnificus (Vieillot, 1817) & Frilled Coquette & OOF,SSF & & \\
\hline Discosura longicaudus (Gmelin, 1788) & Racket-tailed Coquette & OOF & & \\
\hline Chlorestes notata (Reich, 1793) & Blue-chinned Sapphire & OOF,DOF,SSF,ETZ,PF & & \\
\hline
\end{tabular}

Endangered; EN - Endangered; VU - Vulnerable. 
Table 1. Continued...

\begin{tabular}{|c|c|c|c|c|}
\hline \multirow[t]{2}{*}{ Taxon } & \multirow[t]{2}{*}{ English Name } & \multirow[t]{2}{*}{ Vegetation type } & \multicolumn{2}{|c|}{$\begin{array}{l}\text { Category } \\
\text { of threat }\end{array}$} \\
\hline & & & ММА & IUCN \\
\hline Thalurania watertonii (Bourcier, 1847) & Long-tailed Woodnymph & OOF,DOF,SSF,ETZ & EN & EN \\
\hline Hylocharis sapphirina (Gmelin, 1788) & Ruphous-throated Sapphire & OOF,DOF, SSF & & \\
\hline Hylocharis cyanus (Vieillot, 1818) & White-chinned Sapphire & OOF,DOF,SSF & & \\
\hline Heliothryx auritus (Gmelin, 1788) & Black-eared Fairy & OOF,DOF,SSF,ETZ & & \\
\hline $\begin{array}{l}\text { Heliomaster squamosus } \\
\text { (Temminck, 1823) }\end{array}$ & Stripe-breasted Starthroat & OOF,SSF & & \\
\hline Calliphlox amethystina (Bodaert, 1783) & Amethyst Woodstar & OOF,SSF & & \\
\hline Trogon viridis Linnaeus, 1766 & White-tailed Trogon & OOF,DOF,SSF & & \\
\hline Trogon curucui Linnaeus, 1766 & Blue-crowned Trogon & OOF,DOF,SSF,ETZ,PF & & \\
\hline Trogon rufus Gmelin, 1788 & Black-throated Trogon & $\mathrm{OOF}$ & & \\
\hline $\begin{array}{l}\text { Momotus momota marcgravianus } \\
\text { Pinto \& Camargo, } 1961\end{array}$ & Amazonian Motmot & OOF,DOF,SSF,ETZ & EN & \\
\hline Galbula ruficauda Cuvier, 1816 & Rufous-tailed Jacamar & OOF,DOF,SSF,ETZ,PF & & \\
\hline $\begin{array}{l}\text { Ramphastos vitellinus ariel } \\
\text { Vigors, } 1826\end{array}$ & Channel-billed Toucan & OOF,DOF,SSF,ETZ & & EN \\
\hline Pteroglossus inscriptus Swainson, 1822 & Lettered Aracari & OOF,DOF,SSF,ETZ & & \\
\hline Pteroglossus aracari (Linnaeus, 1758) & Black-nacked Aracari & OOF,DOF,SSF & & \\
\hline $\begin{array}{l}\text { Picumnus pernambucensis } \\
\text { Zimmer, } 1947\end{array}$ & Pernambuco Piculet & OOF,DOF,SSF,ETZ,PF & & \\
\hline Veniliornis affinis (Swainson, 1821) & Red-stained Woodpecker & OOF,DOF,SSF,ETZ & & \\
\hline Piculus flavigula (Bodaert, 1783) & Yellow-throated Woodpecker & OOF,DOF,SSF & & \\
\hline Piculus chrysochloros (Vieillot, 1818) & Golden-green Woodpecker & OOF,DOF & & \\
\hline Micrastur ruficollis (Vieillot, 1817) & Barred Forest-Falcon & OOF,DOF,SSF,ETZ & & \\
\hline $\begin{array}{l}\text { Micrastur semitorquatus } \\
\text { (Vieillot, 1817) }\end{array}$ & Collared Forest-Falcon & OOF,DOF,SSF,ETZ & & \\
\hline Brotogeris tirica (Gmelin, 1788) & Plain Parakeet & OOF,DOF,SSF & & \\
\hline Touit surdus (Kuhl, 1820) & Golden-tailed Parrotlet & OOF,DOF,SSF,ETZ & VU & VU \\
\hline Pionus reichenowi Heine, 1844 & Blue-headed Parrot & OOF,DOF,SSF & VU & \\
\hline Pionus maximiliani (Kuhl, 1820) & Scaly-headed Parrot & OOF,DOF & & \\
\hline Amazona amazonica (Linnaeus, 1766) & Orange-winged Parrot & OOF,DOF,SSF,ETZ & & \\
\hline $\begin{array}{l}\text { Terenura sicki } \\
\text { Teixeira \& Gonzaga, } 1983\end{array}$ & Orange-bellied Antwren & OOF,DOF,SSF,ETZ & $\mathrm{CR}$ & EN \\
\hline Myrmotherula axillaris (Vieillot, 1817) & White-flanked Antwren & OOF,DOF,SSF,ETZ,PF & & \\
\hline $\begin{array}{l}\text { Myrmotherula snowi Teixeira \& } \\
\text { Gonzaga, } 1985\end{array}$ & Alagoas Antwren & OOF,SSF,ETZ & $\mathrm{CR}$ & $\mathrm{CR}$ \\
\hline $\begin{array}{l}\text { Thamnomanes caesius caesius } \\
\text { (Temminck, 1820) }\end{array}$ & Cinereous Antshrike & OOF,DOF,SSF & VU & \\
\hline $\begin{array}{l}\text { Dysithamnus mentalis } \\
\text { (Temminck, 1820) }\end{array}$ & Plain Antvireo & OOF,DOF,SSF,ETZ & & \\
\hline $\begin{array}{l}\text { Herpsilochmus atricapillus Pelzeln, } \\
1868\end{array}$ & Black-capped Antwren & OOF,DOF,SSF,ETZ,PF & & \\
\hline Herpsilochmus pectoralis Sclater, 1857 & Pectoral Antwren & SEMIDEPENDENT & & VU \\
\hline
\end{tabular}


Table 1. Continued...

\begin{tabular}{|c|c|c|c|c|}
\hline \multirow[t]{2}{*}{ Taxon } & \multirow[t]{2}{*}{ English Name } & \multirow[t]{2}{*}{ Vegetation type } & \multicolumn{2}{|c|}{$\begin{array}{l}\text { Category } \\
\text { of threat }\end{array}$} \\
\hline & & & ММА & IUCN \\
\hline $\begin{array}{l}\text { Herpsilochmus rufimarginatus } \\
\text { Vieillot, } 1816\end{array}$ & Rufous-winged Antwren & OOF,DOF,SSF,ETZ & & \\
\hline Thamnophilus pelzelni Hellmayr, 1924 & Planalto Slaty-Antshrike & OOF,DOF,SSF,ETZ,PF & & \\
\hline $\begin{array}{l}\text { Thamnophilus caerulescens } \\
\text { pernambucensis Naumburg, } 1937\end{array}$ & Variable Antshrike & OOF,DOF,SSF,ETZ,PF & VU & \\
\hline $\begin{array}{l}\text { Thamnophilus aethiops distans } \\
\text { Pinto, } 1954\end{array}$ & White-shouldered Antshrike & OOF,DOF,SSF,ETZ & $\mathrm{EN}$ & \\
\hline Hypoedaleus guttatus (Vieillot, 1816) & Spot-backed Antshrike & OOF & & \\
\hline $\begin{array}{l}\text { Myrmoderus ruficauda soror } \\
\text { (Pinto, 1940) }\end{array}$ & Scalloped Antbird & OOF,DOF,SSF,ETZ & EN & EN \\
\hline $\begin{array}{l}\text { Pyriglena pernambucensis Zimmer, } \\
1931\end{array}$ & Pernambuco Fire-eye & OOF,DOF,SSF,ETZ & VU & \\
\hline $\begin{array}{l}\text { Cercomacroides laeta sabinoi } \\
\text { (Pinto, 1939) }\end{array}$ & Willis's Antbird & OOF,DOF,SSF,ETZ & & \\
\hline $\begin{array}{l}\text { Drymophila squamata } \\
\text { (Lichtenstein, 1823) }\end{array}$ & Scaled Antbird & OOF,SSF & & \\
\hline Conopophaga cearae Cory, 1916 & Ceara Gnateater & OOF,DOF,SSF,ETZ,PF & EN & \\
\hline $\begin{array}{l}\text { Conopophaga melanops nigrifrons } \\
\text { Pinto, } 1954\end{array}$ & Black-cheeked Gnateater & OOF,DOF,SSF,ETZ & VU & \\
\hline Formicarius colma Boddaert, 1783 & Rufous-capped Antthrush & OOF,DOF,SSF,ETZ & & \\
\hline $\begin{array}{l}\text { Chamaeza campanisona } \\
\text { (Lichtenstein, 1823) }\end{array}$ & Short-tailed Antthrush & SSF & & \\
\hline $\begin{array}{l}\text { Sclerurus macconnelli bahiae } \\
\text { Chubb, } 1919\end{array}$ & Tawny-throated Leaftosser & SSF,ETZ & VU & \\
\hline $\begin{array}{l}\text { Sclerurus caudacutus calligineus } \\
\text { Pinto, } 1954\end{array}$ & Black-tailed Leaftosser & $\mathrm{OOF}$ & $\mathrm{CR}$ & \\
\hline Dendrocincla taunayi Pinto, 1939 & Pernambuco Woodcreeper & OOF,DOF,SSF,ETZ & EN & \\
\hline $\begin{array}{l}\text { Sittasomus griseicapillus (Vieillot, } \\
1816 \text { ) }\end{array}$ & Olivaceous Woodcreeper & OOF,DOF,SSF,ETZ,PF & & \\
\hline Xiphorhynchus atlanticus (Cory, 1916) & Northern Lesser Woodcreeper & OOF,DOF,SSF,ETZ & $\mathrm{VU}$ & \\
\hline $\begin{array}{l}\text { Xiphorhynchus guttatus } \\
\text { (Lichtenstein, 1820) }\end{array}$ & Buff-throated Woodcreeper & OOF,DOF,SSF,ETZ & & \\
\hline $\begin{array}{l}\text { Campylorhamphus trochilirostris } \\
\text { trochilirostris (Lichtenstein, 1820) }\end{array}$ & Red-billed Scythebill & OOF & EN & \\
\hline Dendrocolaptes medius (Todd, 1920) & Todd's Woodcreeper & OOF & EN & \\
\hline Dendrocolaptes platyrostris Spix, 1825 & Planalto Woodcreeper & OOF & & \\
\hline $\begin{array}{l}\text { Xenops minutus alagoanus } \\
\text { Pinto, } 1954\end{array}$ & Plain Xenops & OOF,DOF,SSF,ETZ & VU & \\
\hline Xenops rutilans Temmincki, 1821 & Streaked Xenops & OOF,DOF,SSF,ETZ & & \\
\hline Automolus lammi Zimmer, 1947 & Pernambuco Foliage-Gleaner & OOF,DOF,SSF & EN & $\mathrm{VU}$ \\
\hline $\begin{array}{l}\text { Philydor novaesi } \\
\text { Teixeira \& Gonzaga, } 1983\end{array}$ & Alagoas Foliage-Gleaner & OOF,SSF & EX & $\mathrm{CR}$ \\
\hline $\begin{array}{l}\text { Cichlocolaptes mazarbarnetti } \\
\text { (Mazar-Barnett \& Buzzetti, 2014) }\end{array}$ & Cryptic Treehunter & OOF,SSF & EX & \\
\hline Synallaxis infuscata Pinto, 1950 & Pinto's Spinetail & OOF,DOF,SSF,ETZ & EN & $\mathrm{EN}$ \\
\hline
\end{tabular}

Endangered; EN - Endangered; VU - Vulnerable. 
Table 1. Continued...

\begin{tabular}{|c|c|c|c|c|}
\hline \multirow[t]{2}{*}{ Taxon } & \multirow[t]{2}{*}{ English Name } & \multirow[t]{2}{*}{ Vegetation type } & \multicolumn{2}{|c|}{$\begin{array}{l}\text { Category } \\
\text { of threat }\end{array}$} \\
\hline & & & ММА & IUCN \\
\hline $\begin{array}{l}\text { Cranioleuca semicinerea } \\
\text { (Reichenbach, 1853) }\end{array}$ & Grey-headed Spinetail & OOF,DOF,SSF,ETZ & & \\
\hline $\begin{array}{l}\text { Neopelma pallescens } \\
\text { (Lafresnaye, } 1853 \text { ) }\end{array}$ & Pale-bellied Tyrant-Manakin & OOF,DOF,SSF,ETZ,PF & & \\
\hline $\begin{array}{l}\text { Ceratopipra rubrocapilla } \\
\text { (Temminck, 1821) }\end{array}$ & Red-headed Manakin & OOF,DOF,SSF,ETZ & & \\
\hline Manacus manacus (Linnaeus, 1766) & White-bearded Manakin & OOF,DOF,SSF,ETZ,PF & & \\
\hline Chiroxiphia pareola (Linnaeus, 1766) & Blue-backed Manakin & OOF,DOF,SSF,ETZ,PF & & \\
\hline Oxyruncus cristatus Swainson, 1821 & Sharpbill & OOF,SSF & & \\
\hline Myiobius barbatus (Gmelin, 1789) & Whiskered Flycatcher & OOF,DOF,SSF,ETZ & & \\
\hline Myiobius atricaudus Lawrence, 1863 & Black-tailed Flycatcher & DOF,SSF,ETZ & & \\
\hline $\begin{array}{l}\text { Schiffornis turdina intermedia } \\
\text { Pinto, } 1954\end{array}$ & Thrush-like Schiffornis & OOF,DOF,SSF,ETZ & VU & \\
\hline $\begin{array}{l}\text { Iodopleura pipra leucopygia } \\
\text { Salvin, } 1885\end{array}$ & Buff-throated Purpletuft & OOF,DOF,SSF,ETZ & CR & \\
\hline Tityra inquisitor (Lichtenstein, 1823) & Black-crowned Tityra & OOF & & \\
\hline Tityra cayana (Linnaeus, 1766) & Black-tailed Tityra & OOF,DOF,SSF & & \\
\hline $\begin{array}{l}\text { Pachyramphus marginatus } \\
\text { (Lichtenstein, 1823) }\end{array}$ & Black-capped Becard & OOF,DOF,SSF & & \\
\hline $\begin{array}{l}\text { Pachyramphus validus } \\
\text { (Lichtenstein, 1823) }\end{array}$ & Crested Becard & OOF,SSF & & \\
\hline Lipaugus vociferans (Wied, 1820) & Screaming Piha & OOF,DOF & & \\
\hline Xipholena atropurpurea (Wied, 1820) & White-winged Cotinga & OOF,DOF,SSF,ETZ & VU & EN \\
\hline Procnias averano (Hermann, 1783) & Bearded Bellbird & OOF,SSF,ETZ & & \\
\hline Procnias nudicollis (Vieillot, 1817) & Bare-throated Bellbird & OOF & & VU \\
\hline Carpornis melanocephala (Wied, 1820) & Black-headed Berryeater & OOF & VU & VU \\
\hline Piprites chloris (Temminck, 1822) & Wing-barried Piprites & DOF & & \\
\hline $\begin{array}{l}\text { Platyrinchus mystaceus niveigularis } \\
\text { Pinto, } 1954\end{array}$ & White-throated Spadebill & OOF,DOF,SSF,ETZ & VU & \\
\hline $\begin{array}{l}\text { Mionectes oleagineus } \\
\text { (Lichtenstein, 1823) }\end{array}$ & Ochre-bellied Flycatcher & OOF,DOF,SSF,ETZ & & \\
\hline $\begin{array}{l}\text { Leptopogon amaurocephalus } \\
\text { Tschudi, } 1846\end{array}$ & Sepia-capped Bellbird & OOF,DOF,SSF,ETZ,PF & & \\
\hline Corythopis delalandi (Lesson, 1830) & Southern Antpipit & OOF & & \\
\hline Phylloscartes ceciliae Teixeira, 1987 & Alagoas Tyrannulet & OOF,SSF,ETZ & $\mathrm{CR}$ & EN \\
\hline $\begin{array}{l}\text { Rhynchocyclus olivaceus } \\
\text { (Temminck, 1820) }\end{array}$ & Olicaceous Flatbill & OOF,DOF,SSF & & \\
\hline $\begin{array}{l}\text { Tolmomyias poliocephalus } \\
\text { (Taczanowski, 1884) }\end{array}$ & Gray-crowned Flycatcher & OOF,DOF,SSF & & \\
\hline Tolmomyias flaviventris (Wied, 1831) & Yellow-breasted Flycatcher & OOF,DOF,SSF,ETZ,PF & & \\
\hline $\begin{array}{l}\text { Poecilotriccus plumbeiceps } \\
\text { (Lafresnaye, 1846) }\end{array}$ & Ochre-faced Tody-Flycatcher & OOF,SSF & & \\
\hline Myiornis auricularis (Vieillot, 1818) & Eared Pygmy-Tyrant & OOF,SSF & & \\
\hline
\end{tabular}


Table 1. Continued...

\begin{tabular}{|c|c|c|c|c|}
\hline \multirow[t]{2}{*}{ Taxon } & \multirow[t]{2}{*}{ English Name } & \multirow[t]{2}{*}{ Vegetation type } & \multicolumn{2}{|c|}{$\begin{array}{l}\text { Category } \\
\text { of threat }\end{array}$} \\
\hline & & & ММА & IUCN \\
\hline $\begin{array}{l}\text { Hemitriccus griseipectus naumburgae } \\
\text { (Zimmer, 1945) }\end{array}$ & White-bellied Tody-Tyrant & OOF,DOF,SSF,ETZ & VU & \\
\hline $\begin{array}{l}\text { Hemitriccus mirandae (Snethlage, } \\
\text { 1925) }\end{array}$ & Buff-breasted Tody-Tyrant & OOF,DOF,ETZ & VU & VU \\
\hline Ornithion inerme Hartlaub, 1853 & White-lored Tyrannulet & OOF,DOF,SSF,ETZ & & \\
\hline Elaenia mesoleuca (Deppe, 1830) & Olivaceous Elaenia & OOF,DOF,SSF,ETZ & & \\
\hline Myiopagis gaimardii (d’Orbigny, 1839) & Forest Elaenia & OOF,DOF,SSF,ETZ & & \\
\hline Myiopagis caniceps (Swainson, 1835) & Gray Elaenia & OOF,DOF,ETZ & & \\
\hline Myiopagis viridicata (Vieillot, 1817) & Greenish Elaenia & OOF,DOF,SSF & & \\
\hline $\begin{array}{l}\text { Attila spadiceus uropygiatus } \\
\text { (Wied, 1831) }\end{array}$ & Bright-rumped Attila & OOF,DOF,SSF & VU & \\
\hline Legatus leucophaius (Vieillot, 1818) & Piratic Flycatcher & OOF,DOF,SSF,ETZ,PF & & \\
\hline $\begin{array}{l}\text { Myiarchus tuberculifer } \\
\text { (d'Orbigny \& Lafresnaye, 1837) }\end{array}$ & Dusky-capped Flycatcher & OOF,DOF,SSF & & \\
\hline $\begin{array}{l}\text { Rhytipterna simplex } \\
\text { (Lichtenstein, 1823) }\end{array}$ & Grayish Mourner & OOF,DOF,SSF & & \\
\hline Casiornis fuscus Sclater \& Salvin, 1873 & Ash-throated Casiornis & OOF,DOF,SSF & & \\
\hline $\begin{array}{l}\text { Myiodynastes maculatus } \\
\text { (Statius Muller, 1776) }\end{array}$ & Streaked Flycatcher & OOF,DOF,SSF,ETZ,PF & & \\
\hline $\begin{array}{l}\text { Megarynchus pitangua } \\
\text { (Linnaeus, 1766) }\end{array}$ & Boat-billed Flycatcher & OOF,DOF,SSF,ETZ,PF & & \\
\hline Cnemotriccus fuscatus (Wied, 1831) & Fuscous Flycatcher & OOF,DOF,SSF,ETZ,PF & & \\
\hline Lathrotriccus euleri (Cabanis, 1868) & Euler's Flycatcher & OOF,DOF,SSF,ETZ & & \\
\hline Contopus cinereus (Spix, 1825) & Tropical Pewee & OOF,SSF,ETZ & & \\
\hline Vireo chivi (Vieillot, 1817) & Chivi Vireo & OOF,DOF,SSF,ETZ,PF & & \\
\hline $\begin{array}{l}\text { Pheugopedius genibarbis } \\
\text { (Swainson, 1838) }\end{array}$ & Moustached Wren & OOF,DOF,SSF,ETZ,PF & & \\
\hline $\begin{array}{l}\text { Cantorchilus longirostris } \\
\text { (Vieillot, 1819) }\end{array}$ & Long-billed Wren & OOF,DOF,SSF,ETZ,PF & & \\
\hline $\begin{array}{l}\text { Ramphocaenus melanurus } \\
\text { Vieillot, } 1819\end{array}$ & Long-billed Gnatwren & OOF,DOF,SSF,ETZ,PF & & \\
\hline Turdus flavipes Vieillot, 1818 & Yellow-legged Thrush & OOF,DOF,SSF,ETZ & & \\
\hline Turdus fumigatus Lichtenstein, 1823 & Cocoa Thrush & OOF,DOF,SSF & & \\
\hline Turdus albicollis Vieillot, 1818 & White-necked Thrush & OOF,DOF,SSF,ETZ & & \\
\hline Arremon taciturnus (Hermann, 1783) & Pectoral Sparrow & OOF,DOF,SSF,ETZ,PF & & \\
\hline Setophaga pitiayumi (Vieillot, 1817) & Tropical Parula & OOF,DOF,SSF,ETZ & & \\
\hline $\begin{array}{l}\text { Geothlypis aequinoctialis } \\
\text { (Gmelin, 1789) }\end{array}$ & Masked Yellowthroat & OOF,SSF,PF & & \\
\hline Basileuterus culicivorus (Deppe, 1830) & Golden-crowned Warbler & OOF,DOF,SSF,ETZ,PF & & \\
\hline Myiothlypis flaveola Baird, 1865 & Flavescent Warbler & OOF,DOF,SSF,ETZ,PF & & \\
\hline Anumara forbesi (Sclater, 1886) & Forbes's Blackbird & SEMIDEPENDENT & VU & $\mathrm{EN}$ \\
\hline Saltator maximus (Statius Muller, 1776) & Buff-throated Saltator & OOF,DOF,SSF,ETZ & & \\
\hline
\end{tabular}


Table 1. Continued...

\begin{tabular}{|c|c|c|c|c|}
\hline \multirow[t]{2}{*}{ Taxon } & \multirow[t]{2}{*}{ English Name } & \multirow[t]{2}{*}{ Vegetation type } & \multicolumn{2}{|c|}{$\begin{array}{l}\text { Category } \\
\text { of threat }\end{array}$} \\
\hline & & & ММА & IUCN \\
\hline Saltator fuliginosus (Daudin, 1800) & Black-throated Grosbeak & OOF,DOF,SSF & & \\
\hline Ramphocelus bresilius (Linnaeus, 1766) & Brazilian Tanager & OOF,DOF,SSF,ETZ & & \\
\hline Lanio cristatus (Linnaeus, 1766) & Flame-crested Tanager & OOF,DOF,SSF,ETZ,PF & & \\
\hline Tangara cyanomelas (Wied, 1830) & Silver-breasted Tanager & OOF,DOF,SSF & & \\
\hline Tangara fastuosa (Lesson, 1831) & Seven-colored Tanager & OOF,DOF,SSF,ETZ & $\mathrm{VU}$ & $\mathrm{VU}$ \\
\hline $\begin{array}{l}\text { Tangara cyanocephala } \\
\text { (Statius Muller, 1776) }\end{array}$ & Red-necked Tanager & OOF,DOF,SSF,ETZ & & \\
\hline Cissopis leverianus (Gmelin, 1788) & Magpie Tanager & DOF & & \\
\hline Tersina viridis (Illiger, 1811) & Swallow Tanager & OOF,DOF,SSF,ETZ,PF & & \\
\hline Dacnis cayana (Linnaeus, 1766) & Blue Dacnis & OOF,DOF,SSF,ETZ,PF & & \\
\hline Cyanerpes cyaneus (Linnaeus, 1766) & Red-legged Honeycreeper & OOF,DOF,SSF,ETZ,PF & & \\
\hline Chlorophanes spiza (Linnaeus, 1758) & Green Honeycreeper & OOF,DOF,SSF,ETZ & & \\
\hline Hemithraupis guira (Linnaeus, 1766) & Guira Tanager & OOF,DOF,SSF,ETZ & & \\
\hline $\begin{array}{l}\text { Hemithraupis flavicollis melanoxantha } \\
\text { (Liechtenstein, 1823) }\end{array}$ & Yellow-backed Tanager & OOF,DOF,SSF,ETZ & & \\
\hline $\begin{array}{l}\text { Conirostrum speciosum } \\
\text { (Temminck, 1824) }\end{array}$ & Chestnut-vented Conebill & OOF,DOF,SSF,ETZ & & \\
\hline Tiaris fuliginosus (Wied, 1830) & Sooty Grassquit & OOF,DOF,SSF,ETZ & & \\
\hline Habia rubica (Vieillot, 1817) & Red-crowned Ant-Tanager & OOF,DOF,SSF & & \\
\hline $\begin{array}{l}\text { Caryothraustes canadensis frontalis } \\
\text { (Hellmayr, 1905) }\end{array}$ & Yellow-green Grosbeak & OOF,DOF,SSF & EN & \\
\hline Spinus yarrellii (Audubon, 1839) & Yellow-faced Siskin & SEMIDEPENDENT & VU & VU \\
\hline Euphonia cyanocephala (Vieillot, 1818) & Golden-rumped Euphonia & OOF,SSF & & \\
\hline Euphonia pectoralis (Lathan, 1801) & Chestnut-bellied Euphonia & OOF,DOF,SSF,ETZ & & \\
\hline
\end{tabular}

Vegetation types: OOF - Open Ombrophilous Forest; DOF - Dense Ombrophilous Forest; ETZ - Ecological Tension Zone; SSF - Seasonal Semideciduous Forest; and PF - Pioneer Formation. Category of threat: EX - Extict; CR - Critically Endangered; EN - Endangered; VU - Vulnerable.

lower and drier compared to areas further south (Cestaro, 2002; Olmos, 2003), leading to the lower concentration of birds. Another factor that may be affecting the results is the small number of sampling points further north, although the variables mentioned above may be responsible for the larger concentration of the target birds in Pernambuco and Alagoas.

Among all areas, protected or not protected, the ESEC of Murici was the site that presented the greatest concentration of birds from all three groups. The importance of this reserve (and IBA Murici) is already well known, being considered the place with the largest number of threatened birds of the Americas (Wege and Long, 1995; Bencke et al., 2006). Furthermore, Murici also deserves attention by unique bird records that are absent in other parts of the PEC, such as Geotrygon violacea (Temminck, 1809), Trogon rufus Gmelin, 1788, Hypoedaleus guttatus (Vieillot, 1816), Carpornis melanocephala (Wied, 1820), and Corythopis delalandi (Lesson, 1830).
The three Biological Reserves (Saltinho, Pedra Talhada and Guaribas) also play an important role in the conservation of noteworthy birds, such as Leptodon forbesi (Swann, 1922), Touit surdus (Kuhl, 1820), Xipholena atropurpurea (Wied, 1820), and Spinus yarrellii (Audubon, 1839). Moreover, these three biological reserves are inserted in three IBAs (Guadalupe, Mamanguape and Reserva Biológica de Pedra Talhada), confirming the importance of these sites for the conservation of endemic and threatened birds.

The private reserves also are places of great importance for the conservation of endemic and threatened birds in the Atlantic Forest (Oliveira et al., 2010). The RPPN Frei Caneca and the RPPN Pedra D'Anta make up a single forest block, being an area of extreme importance for bird conservation in the Neotropical region (MazarBarnett et al., 2005; Roda, 2006). These two reserves are also inserted into an IBA (Serra do Urubu), and have formerly hosted Philydor novaesi (Teixeira \& Gonzaga, 1983), and Cichlocolaptes mazarbarnetti (Mazar-Barnett 
(a)

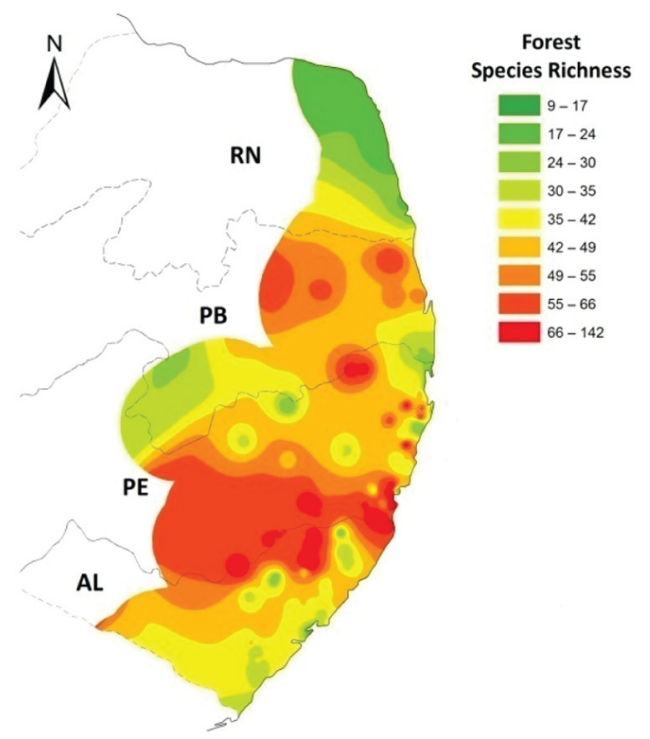

(b)

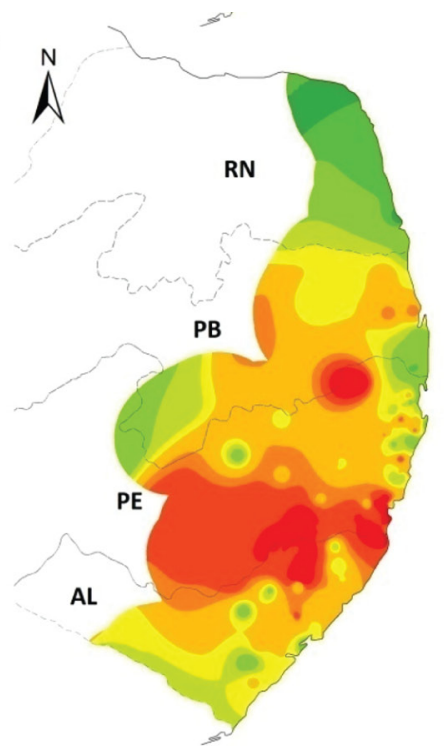

Endemic Species Richness

0-1

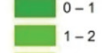

(c)

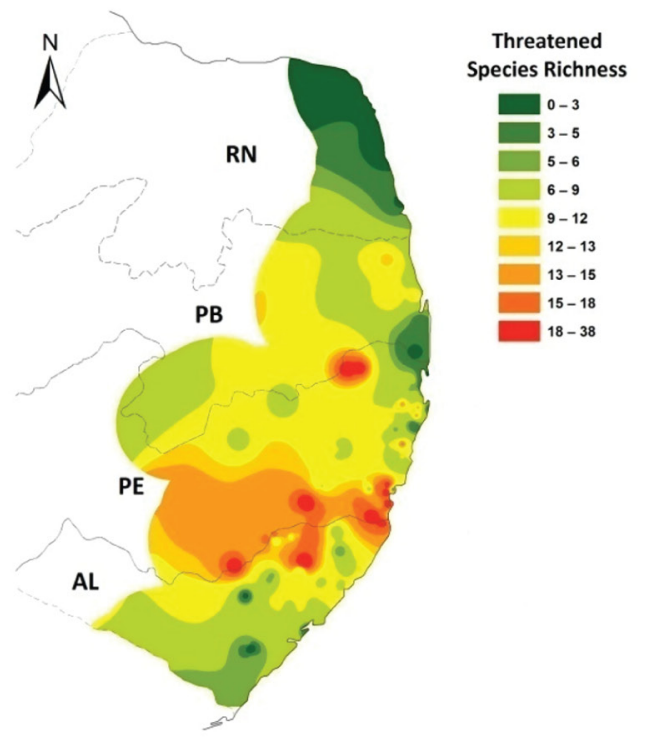

Figure 2. Geographic distribution maps of the total species richness of forest-dependent (a) endemics (b) and threatened birds (c) of the Pernambuco Endemism Center.

\& Buzzetti, 2014), which are now likely to be extinct (Brasil, 2014; Pereira et al., 2014).

Many unprotected forest patches deserve attention in future conservation plans in the region, especially those that were identified with a greater concentration of birds from all three groups, and which are at the same time located in some IBA. Below, we suggest and emphasize that some forest fragments should be turned into protected areas due to two features mentioned earlier:

- The forests of Engenho Coimbra and Mata do Pinto in Alagoas. Both belonging to the Serra Grande mill and are inserted in the IBAs Engenho Coimbra and São José da Laje/Canhotinho, respectively. According to Silveira et al. (2003a), the Engenho
Coimbra comprises one of the most continuous and best-preserved fragments in north-east Brazil. Important birds that can be found there include Penelope superciliaris alagoensis Nardelli, 1993, Terenura sicki (Teixeira \& Gonzaga, 1983), and Odontophorus capueira plumbeicollis (Cory, 1915).

- The forest fragments of Engenho Cachoeira Linda and Mata de Xanguá/Usina Trapiche in south Pernambuco. Both of them are situated within IBA Guadalupe and have important bird records, such as L. forbesi, T. sicki, Anumara forbesi (Sclater, 1886), Myrmoderus ruficauda soror (Pinto, 1940), and $X$. atropurpurea. 
Table 2. Localities in the Pernambuco Endemism Center where ornithological surveys were conducted with their respective geographical coordinates, vegetation types, and species richness of forest-dependent, endemic and/or threatened birds.

\begin{tabular}{|c|c|c|c|c|c|c|}
\hline 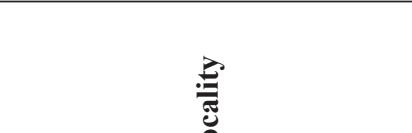 & 氖 & 氖 & : & & 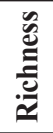 & \\
\hline$\stackrel{0}{\lrcorner}$ & & & & $\mathbf{F}$ & $\mathbf{E}$ & $\mathbf{T}$ \\
\hline PM de Maceió & Maceió (AL) & $9^{\circ} 36^{\prime} \mathrm{S} ; 35^{\circ} 45^{\prime \prime} \mathrm{W}$ & OOF & 28 & 5 & 6 \\
\hline Mata do Catolé & Satuba/Maceió (AL) & $9^{\circ} 33^{\prime} \mathrm{S} ; 35^{\circ} 47^{\prime} \mathrm{W}$ & OOF & 42 & 6 & 7 \\
\hline Mata do Matão & Campo Alegre (AL) & $9^{\circ} 46^{\prime} \mathrm{S} ; 36^{\circ} 14^{\prime} \mathrm{W}$ & OOF & 45 & 7 & 9 \\
\hline Mata de Guardiana-Pitimijú & Cajueiro (AL) & $9^{\circ} 20^{\prime} \mathrm{S} ; 36^{\circ} 09^{\prime} \mathrm{W}$ & OOF & 23 & 4 & 4 \\
\hline $\begin{array}{l}\text { APP Restinga Praia do Francês } \\
\text { e Barra de São Miguel }\end{array}$ & $\begin{array}{l}\text { Barra de São Miguel/Marechal } \\
\text { Deodoro (AL) }\end{array}$ & $9^{\circ} 45^{\prime} \mathrm{S} ; 35^{\circ} 49^{\prime} \mathrm{W}$ & $\mathrm{PF} / \mathrm{OOF}$ & 14 & 1 & 2 \\
\hline Mata da Lagartixa & Cajueiro/Capela (AL) & $9^{\circ} 18^{\prime} \mathrm{S} ; 36^{\circ} 07^{\prime} \mathrm{W}$ & OOF & 24 & 3 & 4 \\
\hline Fazenda Brejo & Teotônio Vilela (AL) & $9^{\circ} 54^{\prime} \mathrm{S} ; 36^{\circ} 18^{\prime} \mathrm{W}$ & SSF & 27 & 0 & 1 \\
\hline RPPN Madeiras & Junqueiro (AL) & $9^{\circ} 51^{\prime} \mathrm{S} ; 36^{\circ} 19^{\prime} \mathrm{W}$ & SSF & 44 & 4 & 6 \\
\hline Usina Santo Antônio I & Barra de Santo Antônio (AL) & $9^{\circ} 23^{\prime} \mathrm{S} ; 35^{\circ} 37^{\prime} \mathrm{W}$ & OOF & 52 & 8 & 12 \\
\hline Usina Santo Antônio II & Barra de Santo Antônio (AL) & $9^{\circ} 23^{\prime} \mathrm{S} ; 35^{\circ} 35^{\prime} \mathrm{W}$ & OOF & 47 & 6 & 8 \\
\hline Mata do Bamburral II & Maceió (AL) & $9^{\circ} 26^{\prime} \mathrm{S} ; 35^{\circ} 41^{\prime} \mathrm{W}$ & OOF & 38 & 8 & 12 \\
\hline $\begin{array}{l}\text { Engenho Coimbra/Usina Serra } \\
\text { Grande }\end{array}$ & Ibateguara (AL) & $9^{\circ} 00^{\prime} \mathrm{S} ; 35^{\circ} 50^{\prime} \mathrm{W}$ & $\mathrm{OOF}$ & 120 & 21 & 31 \\
\hline $\begin{array}{l}\text { Mata do Pinto/Usina Serra } \\
\text { Grande }\end{array}$ & São José da Laje (AL) & $8^{\circ} 58^{\prime} \mathrm{S} ; 36^{\circ} 06^{\prime} \mathrm{W}$ & SSF & 95 & 17 & 22 \\
\hline $\begin{array}{l}\text { Fazenda Riachão/Usina } \\
\text { Coruripe }\end{array}$ & Coruripe (AL) & $10^{\circ} 03^{\prime} \mathrm{S} ; 36^{\circ} 16^{\prime} \mathrm{W}$ & SSF & 31 & 7 & 8 \\
\hline $\begin{array}{l}\text { Mata do Capiatã/Usina } \\
\text { Coruripe }\end{array}$ & Coruripe (AL) & $10^{\circ} 00^{\prime} \mathrm{S} ; 36^{\circ} 16^{\prime} \mathrm{W}$ & SSF & 36 & 5 & 6 \\
\hline $\begin{array}{l}\text { Mata da Sálvia/Usina Utinga } \\
\text { Leão }\end{array}$ & Rio Largo (AL) & $9^{\circ} 32^{\prime} \mathrm{S} ; 35^{\circ} 50^{\prime} \mathrm{W}$ & OOF & 39 & 6 & 11 \\
\hline $\begin{array}{l}\text { Mata do Cedro/Usina Utinga } \\
\text { Leão }\end{array}$ & Rio Largo (AL) & $9^{\circ} 31^{\prime} \mathrm{S} ; 35^{\circ} 54^{\prime} \mathrm{W}$ & $\mathrm{OOF}$ & 45 & 11 & 12 \\
\hline ESEC Murici & Murici/Messias (AL) & $9^{\circ} 12^{\prime} \mathrm{S} ; 35^{\circ} 52^{\prime} \mathrm{W}$ & OOF & 142 & 25 & 39 \\
\hline Mata da Sela/Usina Cachoeira & Flexeiras (AL) & $9^{\circ} 22^{\prime} \mathrm{S} ; 35^{\circ} 43^{\prime} \mathrm{W}$ & OOF & 30 & 6 & 6 \\
\hline $\begin{array}{l}\text { Mata da Encosta do Grotão/ } \\
\text { Usina Camaragibe }\end{array}$ & Matriz de Camaragibe (AL) & $9^{\circ} 06^{\prime} \mathrm{S} ; 35^{\circ} 34^{\prime} \mathrm{W}$ & DOF & 9 & 1 & 1 \\
\hline $\begin{array}{l}\text { Mata de Santa Justina/Usina } \\
\text { Santo Antônio }\end{array}$ & Passo de Camaragibe (AL) & $9^{\circ} 13^{\prime} \mathrm{S} ; 35^{\circ} 30^{\prime} \mathrm{W}$ & $\mathrm{OOF}$ & 44 & 9 & 10 \\
\hline $\begin{array}{l}\text { Grotão do Brás/Mata de Santa } \\
\text { Justina/Usina Santo Antônio }\end{array}$ & Passo de Camaragibe (AL) & $9^{\circ} 13^{\prime} \mathrm{S} ; 35^{\circ} 31^{\prime} \mathrm{W}$ & $\mathrm{OOF}$ & 17 & 4 & 5 \\
\hline RPPN Vila d'Água & Murici (AL) & $9^{\circ} 16^{\prime} \mathrm{S} ; 35^{\circ} 53^{\prime} \mathrm{W}$ & OOF & 31 & 2 & 1 \\
\hline Rebio Pedra Talhada & $\begin{array}{l}\text { Quebrangulo (AL)/Lagoa do } \\
\text { Ouro (PE) }\end{array}$ & $9^{\circ} 14^{\prime} \mathrm{S} ; 36^{\circ} 25^{\prime} \mathrm{W}$ & SSF & 89 & 12 & 20 \\
\hline $\begin{array}{l}\text { Mata do Alto Guzerá/Usina } \\
\text { Serra Grande }\end{array}$ & Ibateguara (AL) & $8^{\circ} 59^{\prime} \mathrm{S} ; 35^{\circ} 58^{\prime} \mathrm{W}$ & SSF & 48 & 8 & 7 \\
\hline $\begin{array}{l}\text { Mata do Apolinário/Usina } \\
\text { Serra Grande }\end{array}$ & São José da Laje (AL) & $8^{\circ} 57^{\prime} \mathrm{S} ; 36^{\circ} 02^{\prime} \mathrm{W}$ & SSF & 50 & 8 & 7 \\
\hline $\begin{array}{l}\text { Mata de Aquibadã/Usina Serra } \\
\text { Grande }\end{array}$ & Ibateguara (AL) & $8^{\circ} 58^{\prime} \mathrm{S} ; 35^{\circ} 54^{\prime} \mathrm{W}$ & $\mathrm{OOF}$ & 57 & 10 & 11 \\
\hline $\begin{array}{l}\text { Mata do Bom Jesus 1/Usina } \\
\text { Serra Grande }\end{array}$ & São José da Laje (AL) & $9^{\circ} 00^{\prime} \mathrm{S} ; 35^{\circ} 50^{\prime} \mathrm{W}$ & SSF & 28 & 2 & 4 \\
\hline $\begin{array}{l}\text { Mata do Bom Jesus 2/Usina } \\
\text { Serra Grande }\end{array}$ & São José da Laje (AL) & $9^{\circ} 00^{\prime} \mathrm{S} ; 36^{\circ} 06^{\prime} \mathrm{W}$ & SSF & 41 & 4 & 6 \\
\hline
\end{tabular}


Table 2. Continued...

\begin{tabular}{|c|c|c|c|c|c|c|}
\hline 芯 & 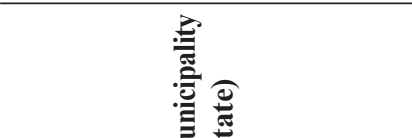 & : & : & & 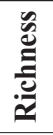 & \\
\hline & $\sum \tilde{E}$ & & & $\mathbf{F}$ & $\mathbf{E}$ & $\mathbf{T}$ \\
\hline $\begin{array}{l}\text { Mata da Cachoeira/Usina Serra } \\
\text { Grande }\end{array}$ & São José da Laje (AL) & $8^{\circ} 56^{\prime} \mathrm{S} ; 36^{\circ} 03^{\prime} \mathrm{W}$ & SSF & 76 & 16 & 18 \\
\hline $\begin{array}{l}\text { Mata do Canivete/Usina Serra } \\
\text { Grande }\end{array}$ & São José da Laje (AL) & $8^{\circ} 57^{\prime} \mathrm{S} ; 36^{\circ} 05^{\prime} \mathrm{W}$ & SSF & 60 & 10 & 12 \\
\hline $\begin{array}{l}\text { Mata do Capoeirão/Usina Serra } \\
\text { Grande }\end{array}$ & São José da Laje (AL) & $8^{\circ} 56^{\prime} \mathrm{S} ; 36^{\circ} 04^{\prime} \mathrm{W}$ & SSF & 58 & 12 & 13 \\
\hline $\begin{array}{l}\text { Mata do Encanamento/Usina } \\
\text { Serra Grande }\end{array}$ & São José da Laje (AL) & $8^{\circ} 57^{\prime} \mathrm{S} ; 36^{\circ} 00^{\prime} \mathrm{W}$ & SSF & 64 & 13 & 15 \\
\hline $\begin{array}{l}\text { Mata do Espinho/Usina Serra } \\
\text { Grande }\end{array}$ & São José da Laje (AL) & $8^{\circ} 57^{\prime} \mathrm{S} ; 36^{\circ} 01^{\prime} \mathrm{W}$ & SSF & 67 & 15 & 15 \\
\hline $\begin{array}{l}\text { Fragmento X/Usina Serra } \\
\text { Grande }\end{array}$ & São José da Laje (AL) & $8^{\circ} 56^{\prime} \mathrm{S} ; 36^{\circ} 02^{\prime} \mathrm{W}$ & SSF & 54 & 10 & 13 \\
\hline $\begin{array}{l}\text { Mata de Ibateguara/Usina Serra } \\
\text { Grande }\end{array}$ & Ibateguara (AL) & $8^{\circ} 57^{\prime} \mathrm{S} ; 35^{\circ} 36^{\prime} \mathrm{W}$ & OOF & 19 & 3 & 3 \\
\hline $\begin{array}{l}\text { Mata do Mal Assombro/Usina } \\
\text { Serra Grande }\end{array}$ & São José da Laje (AL) & $8^{\circ} 58^{\prime} \mathrm{S} ; 36^{\circ} 05^{\prime} \mathrm{W}$ & SSF & 80 & 15 & 17 \\
\hline RPPN Boa Sorte & Murici (AL) & $9^{\circ} 11^{\prime} \mathrm{S} ; 35^{\circ} 55^{\prime} \mathrm{W}$ & OOF & 66 & 13 & 17 \\
\hline RPPN Fazenda São Pedro & Pilar (AL) & $9^{\circ} 33^{\prime} \mathrm{S} ; 35^{\circ} 57^{\prime} \mathrm{W}$ & OOF & 34 & 6 & 7 \\
\hline Fazenda Cachoeira & Pindoba (AL) & $9^{\circ} 28^{\prime} \mathrm{S} ; 36^{\circ} 20^{\prime} \mathrm{W}$ & OOF & 30 & 2 & 2 \\
\hline Rebio Guaribas & Mamanguape/Rio Tinto (PB) & $6^{\circ} 43^{\prime} \mathrm{S} ; 35^{\circ} 11^{\prime} \mathrm{W}$ & $\begin{array}{l}\text { SSF / } \\
\text { ETZ }\end{array}$ & 67 & 7 & 12 \\
\hline Mata da Millenium & Mataraca (PB) & $6^{\circ} 30^{\prime} \mathrm{S} ; 34^{\circ} 58^{\prime} \mathrm{W}$ & SSF & 40 & 3 & 7 \\
\hline RPPN Engenho Gargaú & Santa Rita (PB) & $7^{\circ} 01^{\prime} \mathrm{S} ; 34^{\circ} 57^{\prime} \mathrm{W}$ & SSF & 53 & 9 & 11 \\
\hline PE Mata do Pau Ferro & Areia $(\mathrm{PB})$ & $6^{\circ} 58^{\prime} \mathrm{S} ; 35^{\circ} 44^{\prime} \mathrm{W}$ & OOF & 57 & 5 & 8 \\
\hline RPPN Fazenda Pacatuba & Sapé (PB) & $7^{\circ} 02^{\prime} \mathrm{S} ; 35^{\circ} 09^{\prime} \mathrm{W}$ & SSF & 55 & 9 & 12 \\
\hline Fragmento A/Caaporã & Caaporã (PB) & $7^{\circ} 28^{\prime} \mathrm{S} ; 34^{\circ} 57^{\prime} \mathrm{W}$ & DOF & 33 & 3 & 3 \\
\hline Fragmento B/Caaporã & Caaporã (PB) & $7^{\circ} 28^{\prime} \mathrm{S} ; 34^{\circ} 57^{\prime} \mathrm{W}$ & DOF & 21 & 1 & 2 \\
\hline Fragmento C/Caaporã & Caaporã (PB) & $7^{\circ} 28^{\prime} \mathrm{S} ; 34^{\circ} 57^{\prime} \mathrm{W}$ & DOF & 32 & 1 & 2 \\
\hline Fragmento D/Caaporã & Caaporã (PB) & $7^{\circ} 27^{\prime} \mathrm{S} ; 34^{\circ} 58^{\prime} \mathrm{W}$ & DOF & 32 & 2 & 2 \\
\hline Fragmento E/Caaporã & Caaporã (PB) & $7^{\circ} 27^{\prime} \mathrm{S} ; 34^{\circ} 57^{\prime} \mathrm{W}$ & DOF & 28 & 2 & 4 \\
\hline Fazenda Cidade Viva & Conde (PB) & $7^{\circ} 13^{\prime} \mathrm{S} ; 34^{\circ} 57^{\prime} \mathrm{W}$ & ETZ & 38 & 3 & 3 \\
\hline PE Dois Irmãos & Recife (PE) & $8^{\circ} 00^{\prime} \mathrm{S} ; 34^{\circ} 55^{\prime} \mathrm{W}$ & OOF & 70 & 10 & 14 \\
\hline ESEC Caetés & Paulista (PE) & $7^{\circ} 55^{\prime} \mathrm{S} ; 34^{\circ} 55^{\prime} \mathrm{W}$ & OOF & 61 & 9 & 13 \\
\hline $\begin{array}{l}\text { Refúgio Ecológico Charles } \\
\text { Darwin }\end{array}$ & Igarassu (PE) & $7^{\circ} 48^{\prime} \mathrm{S} ; 34^{\circ} 57^{\prime} \mathrm{W}$ & OOF & 52 & 5 & 7 \\
\hline $\begin{array}{l}\text { Mata do Camocim/ESEC } \\
\text { Tapacurá }\end{array}$ & São Lourenço da Mata (PE) & $8^{\circ} 02^{\prime} \mathrm{S} ; 35^{\circ} 11^{\prime} \mathrm{W}$ & SSF & 65 & 9 & 13 \\
\hline Mata do Toró/ESEC Tapacurá & São Lourenço da Mata (PE) & $8^{\circ} 03^{\prime} \mathrm{S} ; 35^{\circ} 10^{\prime} \mathrm{W}$ & $\begin{array}{l}\mathrm{SSF} / \\
\mathrm{DOF}\end{array}$ & 50 & 5 & 8 \\
\hline RVS Mata do Sistema Gurjaú & Cabo de Santo Agostinho (PE) & $8^{\circ} 13^{\prime} \mathrm{S} ; 35^{\circ} 03^{\prime} \mathrm{W}$ & DOF & 79 & 11 & 17 \\
\hline $\begin{array}{l}\text { Mata do Córrego da Mina/ } \\
\text { Usina São José }\end{array}$ & Igarassu (PE) & $7^{\circ} 45^{\prime} \mathrm{S} ; 35^{\circ} 00^{\prime} \mathrm{W}$ & $\mathrm{OOF}$ & 41 & 6 & 8 \\
\hline $\begin{array}{l}\text { Mata de Zambana/Usina São } \\
\text { José }\end{array}$ & Igarassu (PE) & $7^{\circ} 42^{\prime} \mathrm{S} ; 34^{\circ} 59^{\prime} \mathrm{W}$ & DOF & 53 & 9 & 11 \\
\hline $\begin{array}{l}\text { Mata dos Macacos/Usina São } \\
\text { José }\end{array}$ & Igarassu (PE) & $7^{\circ} 45^{\prime} \mathrm{S} ; 34^{\circ} 59^{\prime} \mathrm{W}$ & OOF & 28 & 1 & 3 \\
\hline
\end{tabular}


Table 2. Continued...

\begin{tabular}{|c|c|c|c|c|c|c|}
\hline : & 䃾 & 氖 & $\stackrel{\Xi}{\stackrel{\Xi}{\leftrightarrows}}$ & & 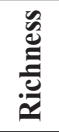 & \\
\hline & & & $\geqslant$ & $\mathbf{F}$ & $\mathbf{E}$ & $\mathbf{T}$ \\
\hline $\begin{array}{l}\text { Mata da Palmeira/Usina São } \\
\text { José }\end{array}$ & Igarassu (PE) & $7^{\circ} 43^{\prime} \mathrm{S} ; 34^{\circ} 59^{\prime} \mathrm{W}$ & $\begin{array}{l}\mathrm{DOF} / \\
\mathrm{OOF}\end{array}$ & 39 & 5 & 7 \\
\hline $\begin{array}{l}\text { Mata de Piedade/Usina São } \\
\text { José }\end{array}$ & Igarassu (PE) & $7^{\circ} 48^{\prime} \mathrm{S} ; 34^{\circ} 59^{\prime} \mathrm{W}$ & OOF & 39 & 6 & 7 \\
\hline RVS Mata do Curado & Recife (PE) & $8^{\circ} 04^{\prime} \mathrm{S} ; 34^{\circ} 57^{\prime} \mathrm{W}$ & $\mathrm{OOF}$ & 33 & 4 & 6 \\
\hline Mata da Ronda & Pombos (PE) & $8^{\circ} 12^{\prime} \mathrm{S} ; 35^{\circ} 22^{\prime} \mathrm{W}$ & SSF & 45 & 8 & 9 \\
\hline PM João Vasconcelos Sobrinho & Caruaru (PE) & $8^{\circ} 22^{\prime} \mathrm{S} ; 36^{\circ} 02^{\prime} \mathrm{W}$ & ETZ & 45 & 7 & 12 \\
\hline Mata do Estado & São Vicente Férrer (PE) & $7^{\circ} 37^{\prime} \mathrm{S} ; 35^{\circ} 30^{\prime} \mathrm{W}$ & ETZ & 77 & 20 & 22 \\
\hline Torre do Microondas & Taquaritinga do Norte (PE) & $7^{\circ} 54^{\prime} \mathrm{S} ; 36^{\circ} 02^{\prime} \mathrm{W}$ & SSF & 23 & 5 & 4 \\
\hline Jardim Botânico do Recife & Recife (PE) & $8^{\circ} 04^{\prime} \mathrm{S} ; 34^{\circ} 58^{\prime} \mathrm{W}$ & $\mathrm{OOF}$ & 19 & 2 & 3 \\
\hline Mata do CMNE & Recife (PE) & $8^{\circ} 04^{\prime} \mathrm{S} ; 34^{\circ} 58^{\prime} \mathrm{W}$ & OOF & 14 & 1 & 2 \\
\hline REBIO Saltinho & Tamandaré/Rio Formoso (PE) & $8^{\circ} 43^{\prime} \mathrm{S} ; 35^{\circ} 10^{\prime} \mathrm{W}$ & DOF & 106 & 18 & 26 \\
\hline $\begin{array}{l}\text { Mata de Maria Maior/Usina } \\
\text { Serra Grande }\end{array}$ & Canhotinho (PE) & $9^{\circ} 01^{\prime} \mathrm{S} ; 36^{\circ} 10^{\prime} \mathrm{W}$ & SSF & 86 & 15 & 19 \\
\hline Mata da Gia & Barreiros (PE) & $8^{\circ} 49^{\prime} \mathrm{S} ; 35^{\circ} 08^{\prime} \mathrm{W}$ & DOF & 34 & 2 & 5 \\
\hline $\begin{array}{l}\text { RPPN Fazenda Santa Beatriz } \\
\text { do Carnijó }\end{array}$ & Moreno (PE) & $8^{\circ} 08^{\prime} \mathrm{S} ; 35^{\circ} 04^{\prime} \mathrm{W}$ & DOF & 30 & 4 & 4 \\
\hline Engenho Nabuco & Maraial (PE) & $8^{\circ} 47^{\prime} \mathrm{S} ; 35^{\circ} 45^{\prime} \mathrm{W}$ & OOF & 59 & 10 & 15 \\
\hline Engenho Gigante & Maraial (PE) & $8^{\circ} 47^{\prime} \mathrm{S} ; 35^{\circ} 46^{\prime} \mathrm{W}$ & $\mathrm{OOF}$ & 71 & 13 & 19 \\
\hline RVS Mata do Amparo & Itamaracá (PE) & $7^{\circ} 46^{\prime} \mathrm{S} ; 34^{\circ} 51^{\prime} \mathrm{W}$ & $\mathrm{DOF} / \mathrm{PF}$ & 30 & 4 & 3 \\
\hline $\begin{array}{l}\text { RVS Mata do Engenho São } \\
\text { João }\end{array}$ & Itamaracá (PE) & $7^{\circ} 45^{\prime} \mathrm{S} ; 34^{\circ} 52^{\prime} \mathrm{W}$ & $\mathrm{DOF} / \mathrm{PF}$ & 19 & 2 & 1 \\
\hline RVS Mata Engenho Macaxeira & Itamaracá (PE) & $7^{\circ} 44^{\prime} \mathrm{S} ; 34^{\circ} 51^{\prime} \mathrm{W}$ & $\mathrm{DOF} / \mathrm{PF}$ & 15 & 0 & 0 \\
\hline RVS Mata Lanço dos Cações & Itamaracá (PE) & $7^{\circ} 42^{\prime} \mathrm{S} ; 34^{\circ} 50^{\prime} \mathrm{W}$ & $\mathrm{DOF} / \mathrm{PF}$ & 14 & 0 & 0 \\
\hline RVS Mata de Santa Cruz & Itamaracá (PE) & $7^{\circ} 42^{\prime} \mathrm{S} ; 34^{\circ} 51^{\prime} \mathrm{W}$ & $\mathrm{DOF} / \mathrm{PF}$ & 15 & 1 & 1 \\
\hline RVS Mata do Jaguaribe & Itamaracá (PE) & $7^{\circ} 44^{\prime} \mathrm{S} ; 34^{\circ} 51^{\prime} \mathrm{W}$ & DOF/PF & 16 & 1 & 1 \\
\hline $\begin{array}{l}\text { RPPN Frei Caneca/RPPN } \\
\text { Pedra D'Anta }\end{array}$ & $\begin{array}{l}\text { Jaqueira/Lagoa dos Gatos } \\
(\mathrm{PE})\end{array}$ & $8^{\circ} 43^{\prime} \mathrm{S} ; 35^{\circ} 50^{\prime} \mathrm{W}$ & $\begin{array}{l}\mathrm{SSF} / \\
\mathrm{OOF}\end{array}$ & 120 & 21 & 32 \\
\hline $\begin{array}{l}\text { Mata de Xanguá/Usina } \\
\text { Trapiche }\end{array}$ & Rio Formoso (PE) & $8^{\circ} 37^{\prime} \mathrm{S} ; 35^{\circ} 11^{\prime} \mathrm{W}$ & DOF & 108 & 19 & 28 \\
\hline $\begin{array}{l}\text { Mata do Benedito/Engenho } \\
\text { Jussará }\end{array}$ & Gravatá $(\mathrm{PE})$ & $8^{\circ} 17^{\prime} \mathrm{S} ; 35^{\circ} 35^{\prime} \mathrm{W}$ & SSF & 56 & 13 & 15 \\
\hline $\begin{array}{l}\text { Mata do Jussará/Engenho } \\
\text { Jussará }\end{array}$ & Gravatá $(\mathrm{PE})$ & $8^{\circ} 18^{\prime} \mathrm{S} ; 35^{\circ} 35^{\prime} \mathrm{W}$ & SSF & 27 & 4 & 5 \\
\hline $\begin{array}{l}\text { Mata do Gringo/Engenho } \\
\text { Jussará }\end{array}$ & Gravatá $(\mathrm{PE})$ & $8^{\circ} 17^{\prime} \mathrm{S} ; 35^{\circ} 34^{\prime} \mathrm{W}$ & SSF & 24 & 4 & 5 \\
\hline Engenho Cachoeira Linda & Barreiros (PE) & $8^{\circ} 49^{\prime} \mathrm{S} ; 35^{\circ} 18^{\prime} \mathrm{W}$ & DOF & 104 & 18 & 25 \\
\hline Engenho Roncadorzinho & Barreiros (PE) & $8^{\circ} 48^{\prime} \mathrm{S} ; 35^{\circ} 17^{\prime} \mathrm{W}$ & DOF & 78 & 14 & 20 \\
\hline Engenho Água Azul & Timbaúba (PE) & $7^{\circ} 36^{\prime} \mathrm{S} ; 35^{\circ} 24^{\prime} \mathrm{W}$ & SSF & 74 & 18 & 22 \\
\hline Mata de Aldeia & $\begin{array}{l}\text { Abreu e Lima/Camaragibe/ } \\
\text { Pau D'alho (PE) }\end{array}$ & $7^{\circ} 54^{\prime} \mathrm{S} ; 35^{\circ} 03^{\prime} \mathrm{W}$ & OOF & 75 & 10 & 13 \\
\hline Mata do CIMNC & $\begin{array}{l}\text { Araçoiaba/Igarassu/Paudalho } \\
\text { (PE) }\end{array}$ & $7^{\circ} 50^{\prime} \mathrm{S} ; 35^{\circ} 07^{\prime} \mathrm{W}$ & $\begin{array}{l}\mathrm{SSF} / \\
\mathrm{OOF}\end{array}$ & 44 & 4 & 7 \\
\hline RPPN Bituri & Brejo da Madre de Deus (PE) & $8^{\circ} 12^{\prime} \mathrm{S} ; 36^{\circ} 23^{\prime} \mathrm{W}$ & SSF & 32 & 3 & 7 \\
\hline Engenho Opinioso & Amaraji (PE) & $8^{\circ} 20^{\prime} \mathrm{S} ; 35^{\circ} 32^{\prime} \mathrm{W}$ & SSF & 38 & 7 & 7 \\
\hline Sítio Vale Verde & Gravatá (PE) & $8^{\circ} 16^{\prime} \mathrm{S} ; 35^{\circ} 33^{\prime} \mathrm{W}$ & SSF & 28 & 4 & 4 \\
\hline
\end{tabular}


Table 2. Continued...

\begin{tabular}{|c|c|c|c|c|c|c|}
\hline \multirow{2}{*}{ 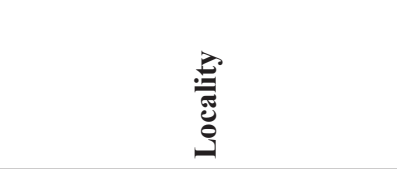 } & \multirow{2}{*}{ 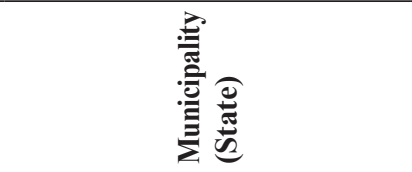 } & \multirow{2}{*}{ 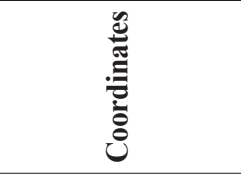 } & \multirow{2}{*}{ 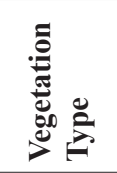 } & \multicolumn{3}{|c|}{ 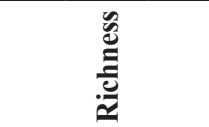 } \\
\hline & & & & $\mathbf{F}$ & $\mathbf{E}$ & $\mathbf{T}$ \\
\hline RPPN Eco Fazenda Morim & $\begin{array}{l}\text { São José da Coroa Grande } \\
\text { (PE) }\end{array}$ & $8^{\circ} 52^{\prime} \mathrm{S} ; 35^{\circ} 13^{\prime} \mathrm{W}$ & DOF & 90 & 16 & 22 \\
\hline $\begin{array}{l}\text { Mata da Cunha/Fazenda } \\
\text { Soberana }\end{array}$ & São Benedito do Sul (PE) & $8^{\circ} 51^{\prime} \mathrm{S} ; 35^{\circ} 54^{\prime} \mathrm{W}$ & $\mathrm{OOF}$ & 52 & 11 & 12 \\
\hline $\begin{array}{l}\text { Grota do Inferno/Engenho } \\
\text { Sacramento }\end{array}$ & Água Preta (PE) & $8^{\circ} 42^{\prime} \mathrm{S} ; 35^{\circ} 24^{\prime} \mathrm{W}$ & DOF & 74 & 12 & 17 \\
\hline $\begin{array}{l}\text { Mata da Ferrugem/Engenho } \\
\text { Sacramento }\end{array}$ & Água Preta (PE) & $8^{\circ} 40^{\prime} \mathrm{S} ; 35^{\circ} 25^{\prime} \mathrm{W}$ & DOF & 56 & 11 & 14 \\
\hline Mata do Dêra/Usina Trapiche & Sirinhaém $(\mathrm{PE})$ & $8^{\circ} 34^{\prime} \mathrm{S} ; 35^{\circ} 10^{\prime} \mathrm{W}$ & DOF & 80 & 14 & 20 \\
\hline $\begin{array}{l}\text { Engenho Jaguaré/Usina } \\
\text { Trapiche }\end{array}$ & Sirinhaém (PE) & $8^{\circ} 33^{\prime} \mathrm{S} ; 35^{\circ} 11^{\prime} \mathrm{W}$ & DOF & 77 & 14 & 18 \\
\hline $\begin{array}{l}\text { Complexo do Jaguarão/Usina } \\
\text { Cucaú }\end{array}$ & Rio Formoso/Sirinhaém (PE) & $8^{\circ} 35^{\prime} \mathrm{S} ; 35^{\circ} 15^{\prime} \mathrm{W}$ & DOF & 68 & 13 & 17 \\
\hline $\begin{array}{l}\text { Mata de Zefa dos Cahorros/ } \\
\text { Usina Cucaú }\end{array}$ & Gameleira (PE) & $8^{\circ} 36^{\prime} \mathrm{S} ; 35^{\circ} 19^{\prime} \mathrm{W}$ & DOF & 29 & 5 & 5 \\
\hline $\begin{array}{l}\text { Mata de Duas Bocas/Usina } \\
\text { Cucaú e Usina Santo André }\end{array}$ & Tamandaré (PE) & $8^{\circ} 43^{\prime} \mathrm{S} ; 35^{\circ} 14^{\prime} \mathrm{W}$ & DOF & 35 & 8 & 10 \\
\hline RFU Mata do Janga & Paulista (PE) & $7^{\circ} 56^{\prime} \mathrm{S} ; 34^{\circ} 50^{\prime} \mathrm{W}$ & $\mathrm{OOF} / \mathrm{PF}$ & 19 & 2 & 1 \\
\hline APA Mata do Engenho Uchôa & Recife (PE) & $8^{\circ} 05^{\prime} \mathrm{S} ; 34^{\circ} 57^{\prime} \mathrm{W}$ & $\mathrm{OOF} / \mathrm{PF}$ & 14 & 2 & 1 \\
\hline $\begin{array}{l}\text { RVS Mata do Engenho } \\
\text { Moreninho }\end{array}$ & Moreno (PE) & $8^{\circ} 06^{\prime} \mathrm{S} ; 35^{\circ} 06^{\prime} \mathrm{W}$ & DOF & 41 & 3 & 4 \\
\hline $\begin{array}{l}\text { Engenho Massaranduba do } \\
\text { Norte }\end{array}$ & Goiana (PE) & $7^{\circ} 36^{\prime} \mathrm{S} ; 34^{\circ} 50^{\prime} \mathrm{W}$ & $\mathrm{DOF} / \mathrm{PF}$ & 22 & 2 & 4 \\
\hline $\begin{array}{l}\text { Engenho Bita/ESEC Bita e } \\
\text { Utinga }\end{array}$ & Ipojuca (PE) & $8^{\circ} 22^{\prime} \mathrm{S} ; 35^{\circ} 03^{\prime} \mathrm{W}$ & DOF & 35 & 3 & 4 \\
\hline $\begin{array}{l}\text { Engenho Utinga/ESEC Bita e } \\
\text { Utinga }\end{array}$ & $\begin{array}{l}\text { Cabo de Santo Agostinho/ } \\
\text { Ipojuca (PE) }\end{array}$ & $8^{\circ} 20^{\prime} \mathrm{S} ; 35^{\circ} 03^{\prime} \mathrm{W}$ & DOF & 48 & 7 & 9 \\
\hline Fragmento 1/Usina Ipojuca & Ipojuca (PE) & $8^{\circ} 22^{\prime} \mathrm{S} ; 35^{\circ} 05^{\prime} \mathrm{W}$ & DOF & 21 & 3 & 3 \\
\hline Fragmento 2/Usina Ipojuca & Ipojuca (PE) & $8^{\circ} 23^{\prime} \mathrm{S} ; 35^{\circ} 06^{\prime} \mathrm{W}$ & DOF & 14 & 1 & 0 \\
\hline Engenho Providência & Cabo de Santo Agostinho (PE) & $8^{\circ} 20^{\prime} \mathrm{S} ; 35^{\circ} 07^{\prime} \mathrm{W}$ & DOF & 37 & 5 & 8 \\
\hline $\begin{array}{l}\text { Mata/Loteamento/Bonito } \\
\text { EcoParque }\end{array}$ & Bonito (PE) & $8^{\circ} 34^{\prime} \mathrm{S} ; 35^{\circ} 43^{\prime} \mathrm{W}$ & $\begin{array}{l}\mathrm{SSF} / \\
\mathrm{ETZ}\end{array}$ & 48 & 6 & 7 \\
\hline PNM Mucuri-Hymalaia & Bonito (PE) & $8^{\circ} 32^{\prime} \mathrm{S} ; 35^{\circ} 43^{\prime} \mathrm{W}$ & $\begin{array}{l}\mathrm{SSF} / \\
\mathrm{ETZ}\end{array}$ & 51 & 8 & 9 \\
\hline Restinga da Praia do Gamela & Sirinhaém $(\mathrm{PE})$ & $8^{\circ} 40^{\prime} \mathrm{S} ; 35^{\circ} 05^{\prime} \mathrm{W}$ & $\mathrm{PF}$ & 10 & 0 & 0 \\
\hline PE Mata de Zumbi & Cabo de Santo Agostinho (PE) & $8^{\circ} 18^{\prime} \mathrm{S} ; 34^{\circ} 59^{\prime} \mathrm{W}$ & DOF & 62 & 9 & 13 \\
\hline PE Mata de Duas Lagoas & Cabo de Santo Agostinho (PE) & $8^{\circ} 18^{\prime} \mathrm{S} ; 34^{\circ} 59^{\prime} \mathrm{W}$ & DOF & 52 & 7 & 8 \\
\hline Mata do Franco & Sirinhaém (PE) & $8^{\circ} 36^{\prime} \mathrm{S} ; 35^{\circ} 08^{\prime} \mathrm{W}$ & DOF & 14 & 3 & 3 \\
\hline Mata do Franco II & Sirinhaém (PE) & $8^{\circ} 36^{\prime} \mathrm{S} ; 35^{\circ} 08^{\prime} \mathrm{W}$ & DOF & 16 & 2 & 2 \\
\hline RPPN Jussaral & Catende (PE) & $8^{\circ} 36^{\prime} \mathrm{S} ; 35^{\circ} 43^{\prime} \mathrm{W}$ & SSF & 52 & 9 & 14 \\
\hline $\begin{array}{l}\text { RPPN Mata Estrela Senador } \\
\text { Antônio Farias }\end{array}$ & Baía Formosa (RN) & $6^{\circ} 22^{\prime} \mathrm{S} ; 35^{\circ} 00^{\prime} \mathrm{W}$ & $\mathrm{SSF} / \mathrm{PF}$ & 28 & 4 & 7 \\
\hline PE Mata da Pipa & Tibau do Sul (RN) & $6^{\circ} 14^{\prime} \mathrm{S} ; 35^{\circ} 03^{\prime} \mathrm{W}$ & $\mathrm{SSF} / \mathrm{PF}$ & 20 & 0 & 2 \\
\hline Parque das Dunas & Natal (RN) & $5^{\circ} 50^{\prime} \mathrm{S} ; 35^{\circ} 11^{\prime} \mathrm{W}$ & $\mathrm{PF}$ & 19 & 1 & 2 \\
\hline
\end{tabular}




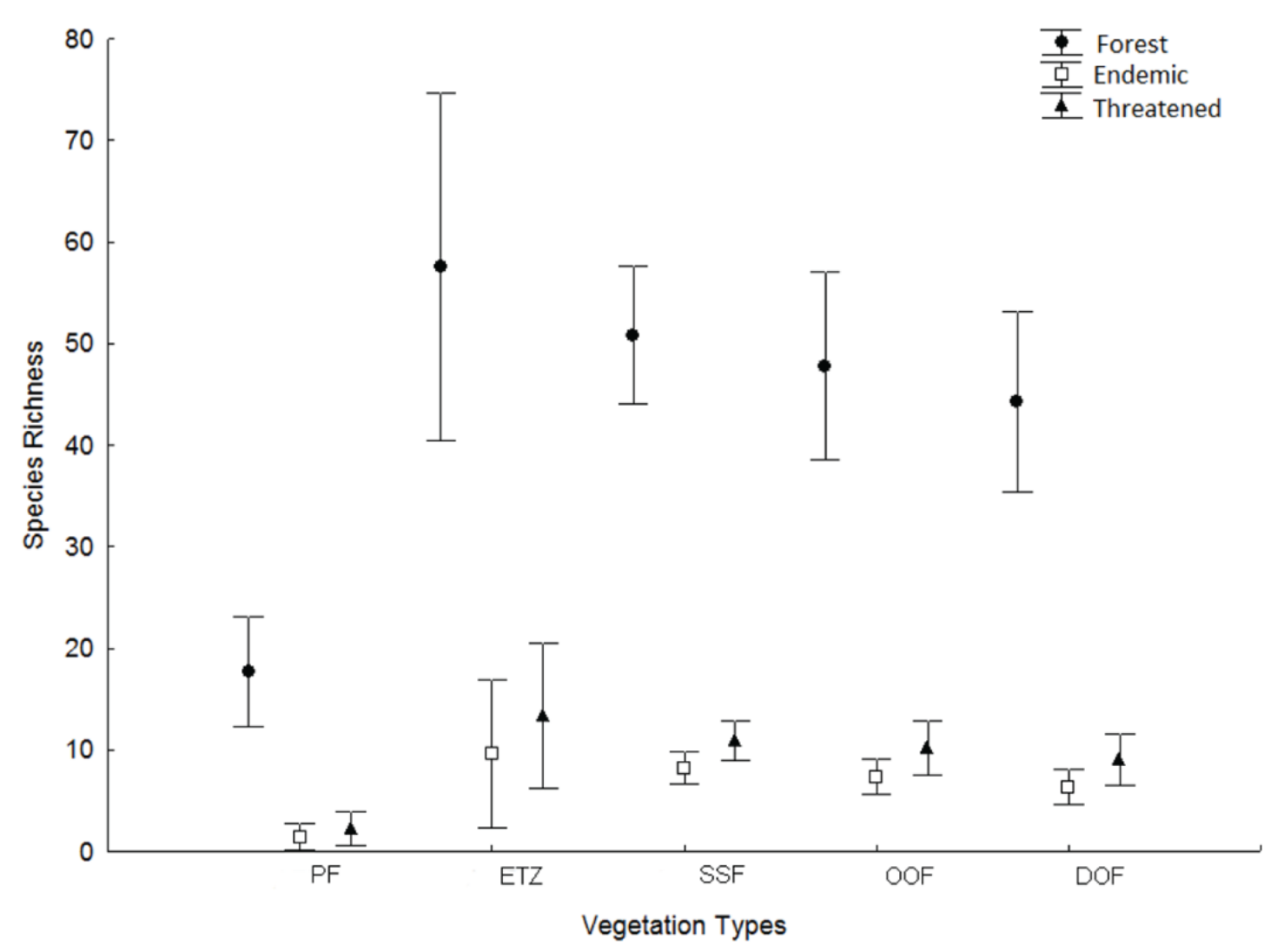

Figure 3. Distribution of the values of species richness of forest, endemic and/or threatened birds in each vegetation type of the Pernambuco Endemism Center (bars indicate $95 \%$ of confidence intervals). Vegetation types: OOF - Open Ombrophilous Forest; DOF - Dense Ombrophilous Forest; ETZ - Ecological Tension Zone; SSF - Seasonal Semideciduous Forest; and $\mathrm{PF}$ - Pioneer Formation.

Table 3. Comparison by ANOVA and $\mathrm{p}$ values of the Tukey test of species richness of forest-dependent, endemic and threatened birds among the vegetation types of the Pernambuco Endemism Center. The values in bold represent the significant differences with an alpha of 0.05 .

\begin{tabular}{|c|c|c|c|c|c|c|c|c|c|c|c|c|}
\hline & \multicolumn{4}{|c|}{ Forest } & \multicolumn{4}{|c|}{ Endemic } & \multicolumn{4}{|c|}{ Threatened } \\
\hline ANOVA & $\mathrm{F}(4 ; 1$ & 7) $=2.5$ & $23 ; \mathbf{p}=$ & 0.024 & $\mathrm{~F}(4 ;$ & 7) $=3$. & $33 ; \mathbf{p}=$ & 0.019 & $\mathrm{~F}(4 ;$ & 7) $=2$, & $47 ; \mathbf{p}=$ & 0.042 \\
\hline Tukey (p) & ETZ & SSF & OOF & DOF & ETZ & SSF & OOF & DOF & ETZ & SSF & OOF & DOF \\
\hline $\mathrm{PF}$ & 0.001 & 0.012 & 0.030 & 0.076 & 0.001 & 0.013 & 0.044 & 0.142 & 0.002 & 0.030 & 0.061 & 0.151 \\
\hline ETZ & & 0.966 & 0.878 & 0.700 & 0.000 & 0.968 & 0.833 & 0.554 & & 0.924 & 0.816 & 0.593 \\
\hline SSF & & & 0.998 & 0.970 & & 0.000 & 0.994 & 0.908 & & & 0.999 & 0.970 \\
\hline OOF & & & & 0.997 & & & & 0.991 & & & & 0.996 \\
\hline
\end{tabular}

Vegetation types: OOF - Open Ombrophilous Forest; DOF - Dense Ombrophilous Forest; ETZ - Ecological Tension Zone; SSF

- Seasonal Semideciduous Forest; and PF - Pioneer Formation.

- The forests in mountainous areas in the municipalities of Bonito and Gravatá. There are still some well-preserved forests; however there are very few protected areas and there is no IBA. There, we can find L. forbesi, Phylloscartes ceciliae (Teixeira, 1987), T. sicki, and Myrmotherula snowi (Teixeira \& Gonzaga, 1985).

We conclude that, despite the "simplicity" of the IDW method, the information on the distribution pattern of the bird groups studied in this work were presented for the first time very clearly, concisely and visually, even when comparing it with previous work concerning the distribution pattern of some birds in the region.

In addition, we showed that some protected areas in this region really ensure the conservation of endemic and threatened birds due to the high density of them and being within the limits of the IBAs. Furthermore, we suggested and emphasized the need for the protection of some places of extreme importance for bird conservation. In this way, we hope that these areas may be analyzed with special 

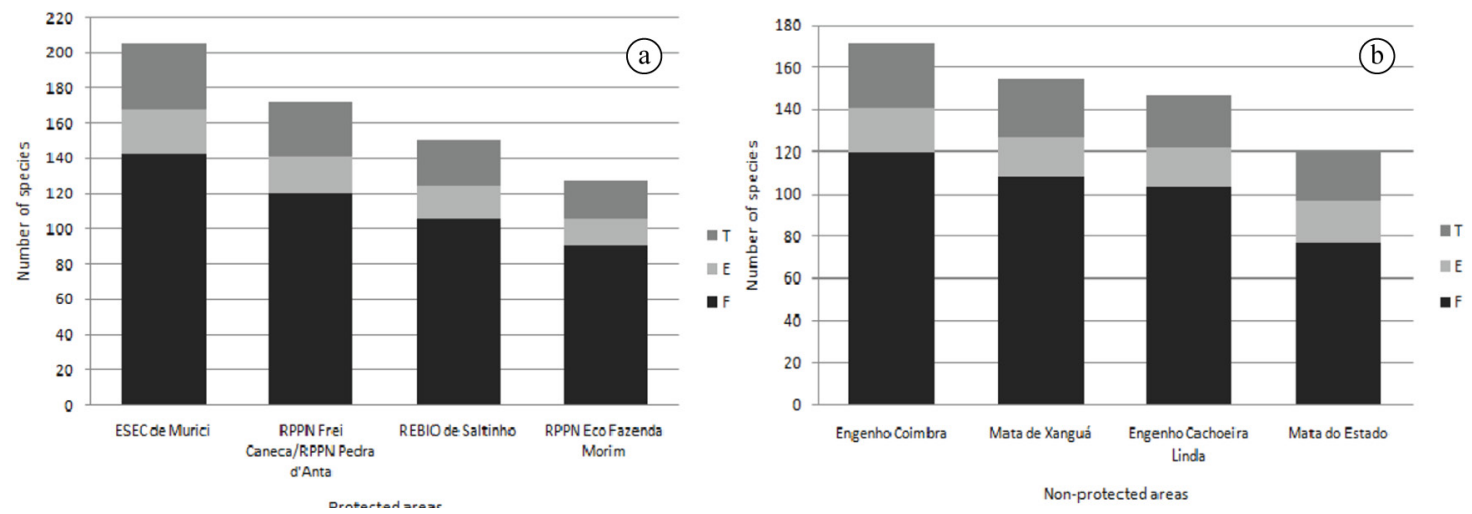

Figure 4. The four protected (a) and non-protected (b) areas with the heighest number of bird species of the three groups in the Pernambuco Endemism Center. T - Threatened with extinction; E - Endemic; and F - Forest-dependent.

attention from now on for the next implementation of protected areas programs in the Atlantic Forest.

\section{Acknowledgements}

We thank the ornithologists Sergio Leal, Galileu Coelho, Ciro Albano, Francisco Sargot Martin, Mauricio Periquito, Frederico Sonntag, Anita Studer and Marcelo Silva for providing some of their bird records and lists. The first author would like to thank CAPES (Coordenação de Aperfeiçoamento de Nível Superior) for providing the scholarship for this study. We also thank the owners and directors of the mills, protected and, non-protected areas that we visited. Thanks to John Medcraft and Alexander Lees for revising the English and for great comments.

\section{References}

ABDI, A. and NANDIPATI, A., 2009. Bird diversity modeling using Geostatistics anf GIS. In: 12th AGILE International Conference on Geographic Information Science, 2-5 June 2009, Hannover, Germany. Hannover: Universitat Hannover, pp. 1-20.

BENCKE, G.A., MAURÍCIO, G.N., DEVELEY, P.F. and GOERCK, J.M., 2006. Áreas Importantes para a conservação das aves no Brasil: parte I - estados do domínio da Mata Atlântica. São Paulo: SAVE Brasil. 494 p.

BRASIL. Ministério do Meio Ambiente-MMA, 2014. Portarias $n^{\circ} 444$ e $n^{\circ} 445$, de 18 de dezembro de 2014. Diário Oficial da Repúbica Federativa do Brasil, Brasília, Seção 1, pp. 121-130.

CESTARO, L.A., 2002. Fragmentos de florestas atlânticas no Rio Grande do Norte: relações estruturais, florísticas e fitogeográficas. São Carlos: Universidade Federal de São Carlos, 149 p. Tese de Doutorado em Ecologia e Recursos Florestais.

CHILDS, C., 2004 [viewed 2015 April 19]. Interpolation surfaces in Arc Gis Spatial Analyst [online]. Redlands: ESRI. Available from: http:// www.esri.com/news/arcuser/0704/files/interpolating.pdf

FUNDAÇÃO SOS MATAATLÂNTICA - SOSMA, 2015 [viewed 2015 August 04]. Atlas dos remanescentes florestais da Mata Atlântica - periodo 2013-2014 [online]. São Paulo: Fundação
SOS Mata Atlântica. Available from: https://www.sosma.org.br/ link/atlas_2013-2014_Mata_Atlantica_relatorio_tecnico_2015.pdf

GIORGI, A.P., ROVZAR, C., DAVIS, K.S., FULLER, T., BUERMANN, W., SAATCHI, S., SMITH, T.B., SILVEIRA, L.F. and GILLESPIE, T.W., 2014. Spatial conservation planning framework for assessing conservation opportunities in the Atlantic Forest of Brazil. Applied Geography (Sevenoaks, England), vol. 53, pp. 69-376. http://dx.doi.org/10.1016/j.apgeog.2014.06.013.

INSTITUTO BRASILEIRO DE GEOGRAFIA E ESTATÍSTICA - IBGE, 1985. Atlas Nacional do Brasil, Região Nordeste. Rio de Janeiro: IBGE.

INSTITUTO BRASILEIRO DE GEOGRAFIA E ESTATÍSTICA - IBGE, 2004. Mapa da vegetação do Brasil: escala 1:5.000.000. 3rd ed. Rio de Janeiro: IBGE.

INTERNATIONAL UNION FOR CONSERVATION OF NATURE - IUCN, 2015 [viewed 2015 August 04]. The IUCN red list of threatened species: version 2015.1 [online]. Cambridge: IUCN. Available from: http://www.iucnredlist.org

KARR, J.R., 1990. Interactions between forest birds and their habitats: a comparative synthesis. In: A. KEAST. Biogeography and ecology of forest bird communities. The Hague: Academic Publishing, pp. 379-386.

LEES, A.C. and PERES, C.A., 2008. Avian life history determinants of local extinction risk in a fragmented Neotropical forest landscape. Animal Conservation, vol. 11, no. 2, pp. 128-137. http://dx.doi. org/10.1111/j.1469-1795.2008.00162.x.

MAZAR-BARNETT, J., CARLOS, C.J. and RODA, S.A., 2005. Renewed hope for the threatened avian endemics of northeastern Brazil. Biodiversity and Conservation, vol. 14, no. 9, pp. 22652274. http://dx.doi.org/10.1007/s10531-004-5290-8.

MOURA, N.G., LEES, A.C., ALEIXO, A., BARLOW, J., DANTAS, S.M., FERREIRA, J., LIMA, M.D.F.C. and GARDNER, T.A., 2014. Two hundred years of local avian extinctions in Eastern Amazonia. Conservation Biology, vol. 28, no. 5, pp. 1271-1281. http://dx.doi.org/10.1111/cobi.12300. PMid:24779443.

MYERS, N., MITTERMEIER, R.A., MITTEMEIER, C.G. and FONSECA, G.A.B., 2000. Biodiversity hotspots for conservation priorities. Nature, vol. 403, no. 6772, pp. 853-858. http://dx.doi. org/10.1038/35002501. PMid:10706275. 
NEWTON, I., 2003. The speciatiobn \& biogeography of birds. San Diego: Academic Press. 668 p.

OLIVEIRA, V.B., PAGLIA, A.P., FONSECA, M. and GUIMARÃES, E., 2010. RPPN e biodiversidade: o papel das reservas particulares na proteção da biodiversidade da Mata Atlântica. São Paulo: Fundação SOS Mata Atlântica. 48 p.

OLMOS, F., 2003. Birds of Mata Estrela private reserve, Rio Grande do Norte, Brazil. Cotinga, n. 20, pp. 26-30.

OLMOS, F., 2005. Aves ameaçadas, prioridades e políticas de conservação no Brasil. Natureza \& Conservação, vol. 3, no. 1, pp. 21-42.

PAULA, L.A., 2012. Cadastro das unidades de conservação. In: B.P.C. BRANCO and M.D.V.C. MELO. Saberes e fazeres da Mata Atlântica do Nordeste: tecendo uma rede de gestores. Recife: AMANE, pp. 69-92.

PEREIRA, G.A., DANTAS, S.M., SILVEIRA, L.F., RODA, A.S., ALBANO, C., SONNTAG, F.A., LEAL, S., PERIQUITO, M.C., MALACCO, G.B. and LEES, A.C., 2014. Status of the globally threatened forest birds of northeast Brazil. Papéis Avulsos de Zoologia, vol. 54, no. 14, pp. 177-194. http://dx.doi. org/10.1590/0031-1049.2014.54.14.

PIACENTINI, V.Q., PIACENTINI, V.Q., ALEIXO, A, AGNE, C.E., MAURICIO, G.N., PACHECO, J.F., BRAVO, G.A., BRITO, G.R.R., NAKA, L.N., OLMOS, F., POSSO, S., SILVEIRA, L.F., BETINI, G.S., CARRANO, E., FRANZ, I., LEES, A.C., LIMA, L.M., PIOLI, D., SCHUNCK, F., AMARAL, F.R., BENCKE, G.A., COHN-HAFT, M., FIGUEIREDO, L.F.A., STRAUBE, F.C. and CESARI, E., 2015. Annotated checklist of the birds of Brazil by the Brazilian Ornithological Records Committee. Revista Brasileira de Ornitologia, vol. 23, no. 2, pp. 91-298.

ROBERTS, E.A., SHELEY, R.L. and LAWRENCE, R.L., 2004. Using sampling and inverse distance weighted modeling for mapping invasive plants. Western North American Naturalist, vol. 64 , no. 3 , pp. 312-323.

RODA, A.S. and PEREIRA, G.A., 2006. Distribuição recente e conservação das aves de rapina florestais do Centro Pernambuco. Revista Brasileira de Ornitologia, vol. 14, no. 4, pp. 331-344.

RODA, S.A., 2003. Aves do Centro de Endemismo Pernambuco: composição, biogeografia e conservação. Belém: Universidade Federal do Pará, 520 p. Tese de Doutorado em Ciências Biológicas.
RODA, S.A., 2006. Aves. In: K.C. PÔRTO, J.S. ALMEIDA-CORTEZ and M. TABARELLI. Diversidade biológica e conservação da Floresta Atlântica ao Norte do Rio São Francisco. Brasília: Ministério do Meio Ambiente, pp. 279-299.

RODA, S.A., PEREIRA, G.A. and ALBANO, C., 2011. Conservação de Aves endemicas e ameaçadas do Centro de Endemismo Pernambuco. Recife: Editora Universitária da UFPE. 79 p.

SILVA, J.M.C., SOUSA, M.C. and CASTELLETI, C.H.M., 2004. Areas of endemism for passerine birds in the Atlantic Forest. Global Ecology and Biogeography, vol. 13, no. 1, pp. 85-92. http://dx.doi.org/10.1111/j.1466-882X.2004.00077.x.

SILVEIRA, L.F., OLMOS, F. and LONG, A., 2003a. Birds in Atlantic Forest Fragments in north-east Brazil. Cotinga, n. 20, pp. 32-46.

SILVEIRA, L.F., OLMOS, F., RODA, S.A. and LONG, A., 2003b. Notes on the seven-coloured Tanager Tangara fastuosa in north-east Brazil. Cotinga, n. 20, pp. 82-88.

SILVEIRA, L.F., RODA, S.A., SANTOS, A.M.M., SOARES, E.S. and BIANCHI, C.A., 2008. Plano de ação para a conservação do mutum-de-alagoas (Mitu mitu = Pauxi mitu). Brasília: ICMBio. 48 p.

SISTEMA NACIONAL DE UNIDADES DE CONSERVAÇÃO - SNUC, 2000. Sistema Nacional de Unidades de conservação: texto da Lei 9.985 de 18 de julho de 2000 e vetos da presidência da República ao PL aprovado pelo congresso Nacional. 2nd ed. São Paulo: Conselho Nacional da Reserva da Biosfera da Mata Atlântica. 76 p.

TABARELLI, M., SIQUEIRA-FILHO, J.A. and SANTOS, A.M.M., 2006. Conservação da Floresta Atlântica ao Norte do Rio São Francisco. In: K.C. PÔRTO, J.S. ALMEIDA-CORTEZ and M. TABARELLI. Biodiversidade Biológica e Conservação da Floresta Atlântica ao Norte do Rio São Francisco. Brasília: Ministério do Meio Ambiente, pp. 41-48.

WEGE, D.C. and LONG, A.J., 1995. Key areas for threatened birds in the Neotropics. Cambridge: BirdLife International. 311 p.

WHITTAKER, R.J., ARAÚJO, M.B., JEPSON, P., LADLE, R.J., WATSON, J.E.M. and WILLIS, K.J., 2005. Conservation biogeography: assessment and prospect. Diversity \& Distributions, vol. 11 , no. 1 , pp. 3-23. http://dx.doi.org/10.1111/j.13669516.2005.00143.x. 